\title{
Visibilité du destin commun et invisibilité de l'histoire : discours, célébrations et construction de la citoyenneté en Nouvelle-Calédonie
}

\section{Stéphanie Graff}

\section{OpenEdition}

\section{Journals}

Édition électronique

URL : http://journals.openedition.org/anthrovision/2250

DOI : 10.4000/anthrovision.2250

ISSN : 2198-6754

\section{Éditeur}

VANEASA - Visual Anthropology Network of European Association of Social Anthropologists

Référence électronique

Stéphanie Graff, «Visibilité du destin commun et invisibilité de l'histoire : discours, célébrations et construction de la citoyenneté en Nouvelle-Calédonie », Anthrovision [En ligne], 4.1 | 2016, mis en ligne le 31 décembre 2016, consulté le 19 avril 2019. URL : http://journals.openedition.org/ anthrovision/2250; DOI : 10.4000/anthrovision.2250

Ce document a été généré automatiquement le 19 avril 2019

(c) Anthrovision 


\title{
Visibilité du destin commun et invisibilité de l'histoire : discours, célébrations et construction de la citoyenneté en Nouvelle-Calédonie
}

\author{
Stéphanie Graff
}

1 Depuis la prise de possession par la France en 1853, la Nouvelle-Calédonie a été marquée par de violents conflits. La source de ces conflits est sans conteste le refus et la résistance du peuple autochtone, le peuple kanak, à se voir soumis à un système dominant ${ }^{2}$. L'administration coloniale lui imposa cependant le cantonnement dans des réserves, le régime de l'indigénat (Merle 1995, 2004). Ce n'est qu'en 1946, avec l'abolition du régime de l'indigénat, que les Kanak devinrent des citoyens français; ils n'obtinrent toutefois le droit de vote qu'en $1957^{3}$.

2 La Nouvelle-Calédonie fut d'abord terre de bagne. Puis, en 1894 le gouverneur Feillet orchestra une politique de colonisation de peuplement libre. Des travailleurs provenant d'autres territoires d'outre-mer français ou étrangers (Ni-Vanuatu, Indonésiens, Vietnamiens, Wallisiens et Futuniens, Polynésiens, Réunionnais, Antillais, Chinois et aujourd'hui Philippins) y furent engagés pour fournir de la main d'œuvre sur les mines et diverses plantations. Dès la fin des années 1940, une politique de colonisation de peuplement fut appliquée par la France, intensifiée sous la présidence de Charles de Gaulle, puis sous le gouvernement Messmer dans les années 19704.

3 En conséquence, le peuple kanak devint progressivement minoritaire sur ses terres, ce qui fut un facteur clef de la revendication indépendantiste et de la cristallisation du débat politique autour de la définition du corps électoral pour un éventuel référendum d'autodétermination ${ }^{5}$. Ce fut un des principaux facteurs déclencheurs des conflits de la période dite des "Evènements ${ }^{6}$ qui prit fin avec la signature des Accords de Matignon en 1988 entre le Front de Libération Nationale Kanak et Socialiste (FLNKS), le Rassemblement Pour la Calédonie dans la République (RPCR) et l'État français 7 . Ces 
accords mettaient en place un nouveau statut d'autonomie pour la Nouvelle-Calédonie et l'engageaient dans un processus de décolonisation qui devait prendre fin avec un scrutin d'autodétermination en 1998. Ce scrutin n'eut pas lieu mais un nouvel accord, l'Accord de Nouméa, fut signé à la place le 5 mai 1998 par les structures précitées. Il prévoit qu’à partir de 2018 une partie de la population devra s'exprimer par vote lors d'une consultation sur l'accession du pays à la pleine souveraineté8. Aujourd'hui encore, la question de la souveraineté et de l'indépendance est au cœur des conflits. De même, la question de la définition du corps électoral reste problématique et fait l'objet, depuis le 1 er mars 2016 d'une mission des Nations Unies chargée d'observer le processus d'établissement et de révision des listes électorales spéciales 9 .

L'application de l'Accord de Nouméa de 1998 a suscité le développement d'une politique mise en œuvre par les pouvoirs publics (locaux calédoniens et étatiques français), celle du "destin commun $»^{10}$. J'analyserai ici les enjeux politiques de la mise en place de ces termes à travers deux exemples : la «fête de la citoyenneté » et «l'affaire des cases ». L'argument de cet article est que cette " politique de destin commun » réécrit l'histoire, en mettant en avant et en rendant visible certains symboles qu'elle mythifie au détriment de l'expression vivante de la culture kanak qui se voit alors davantage marginalisée et stigmatisée. Les deux exemples de la " fête de la citoyenneté » et de « l'affaire des cases » montreront que ce révisionnisme historique et culturel impliqué par la "politique de destin commun » se répercute, dans les politiques publiques, sur ce qu'il est acceptable de voir et de montrer à la société calédonienne au cœur de la ville de Nouméa. Ces deux exemples permettent d'analyser dans l'espace urbain et public calédonien la manière dont l'idéologie et les effets pratiques de l'injonction de la politique de destin commun se manifestent, parfois même de manière brutale pour une partie de la population kanak. L'apport méthodologique du visuel ajoute ici à l'analyse anthropologique textuelle davantage de capacité de représentation, de compréhension, et d'amplitude.

L'objectif de la « politique du destin commun » est double. D'une part, il s'agit de parvenir à une réconciliation entre le peuple et les communautés ${ }^{11}$ en présence sur le territoire. D'autre part, cette politique vise à créer un sentiment d'appartenance à une citoyenneté calédonienne, à faire émerger l'idée d'un "peuple calédonien " partageant une identité commune et une mémoire collective qui unissent les communautés. Cette stratégie politique est basée notamment sur un révisionnisme historique et culturel qui passe par un double mouvement de déplacement et de remplacement des faits, récits, et symboles. Elle tente d'évacuer la question de l'indépendance en soi pour la remplacer par une communauté de destin au sein de laquelle la question de l'indépendance et de la souveraineté du peuple kanak vis-à-vis de la France ne se poserait plus ${ }^{12}$.

6 En effet, depuis la déclaration commune pour l'indépendance élaborée en 1975 par une quarantaine de personnes, membres de l'Union Calédonienne, de l'Union Multiraciale, des Jeunesses Ouvrières Calédoniennes, du Groupe 1878, et des Foulards rouges (Leblic 1993 : 61), les présidents successifs de la République française ne souhaitent pas l'indépendance de la Nouvelle-Calédonie. En outre, si, en 1983, la déclaration de la table ronde de Nainville-les-Roches reconnaît le droit inné et actif du peuple kanak à l'indépendance, le texte précise : « dans le cadre de la Constitution française ${ }^{13}$ ». 


\section{La citoyenneté calédonienne}

7 L'Accord de Nouméa a formulé un « contrat social » notamment dans l'objectif de sceller une citoyenneté calédonienne et d'établir progressivement la notion d'un peuple calédonien. La fusion de toutes les communautés dans une seule communauté de destin, dans un seul "peuple", est donc un principe de base de l'Accord qui est devenu, et depuis la signature de l'Accord en 1998 a été, la principale référence de toutes les politiques et institutions de Nouvelle-Calédonie. Ce principe de "destin commun» trouve ses prémisses dans la déclaration de la table ronde de Nainville-les-Roches lorsque les "victimes de l'histoire» ont été reconnues en tant que tels par les Kanak et les représentants $\mathrm{du}$ Front Indépendantiste ${ }^{14}$. Ce principe retrouve également son incorporation symbolique à travers la poignée de main entre Jacques Lafleur ${ }^{15}$ et JeanMarie Tjibaou ${ }^{16}$, représentant respectivement le RPCR et le FLNKS lors de la signature des Accords de Matignon (1988).

Poignée de main entre Jacques Lafleur (RPCR), à gauche, et Jean-Marie Tjibaou (FLNKS), à droite, après la signature des Accords de Matignon

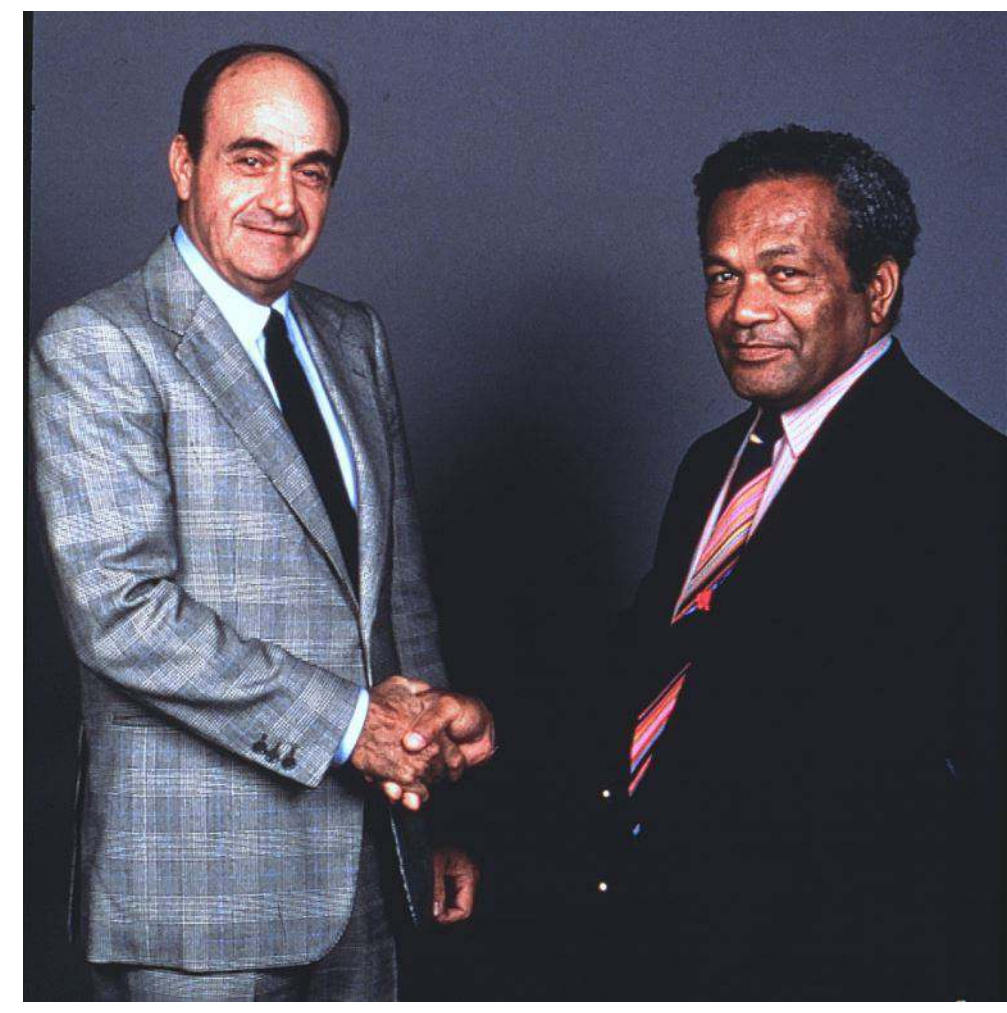

Image 1

(c) ADCK-CCT, G. Merillon \& D. Simon

8 Ainsi, le préambule de l'Accord de Nouméa mentionne: " [qu'il] est aujourd'hui nécessaire de poser les bases d'une citoyenneté de la Nouvelle-Calédonie, permettant au peuple d'origine de se constituer avec les hommes et les femmes qui y vivent une communauté humaine affirmant son destin commun. " S'il faut poser les bases d'une citoyenneté calédonienne, qui sont les citoyens? 
9 Selon Alain Christnacht ${ }^{17}$, « [...] ce qu'on a fait dans l'Accord de Nouméa et qui n'était pas dans les Accords de Matignon, c'est de définir une citoyenneté calédonienne qui est un sous-ensemble de la nation française. C'est-à-dire que tous les citoyens calédoniens ont la nationalité française mais tous les nationaux français vivant en Nouvelle-Calédonie ne sont pas des citoyens calédoniens. Les citoyens calédoniens sont ceux qui sont inclus dans le corps électoral restreint. Les citoyens calédoniens [...] ont deux droits exorbitants que n'ont pas les autres Français vivant en Nouvelle-Calédonie : ils ont le droit de vote pour le scrutin d'autodétermination futur et pour les élections aux assemblées de province et au congrès et ils ont un droit prioritaire à l'emploi ${ }^{18}$. »

10 Les citoyens calédoniens seraient donc les individus inclus dans les corps électoraux restreints pouvant voter aux élections provinciales et à la consultation sur l'accession du pays à la pleine souveraineté prévue à partir de $2018^{19}$.

\section{La « fête de la citoyenneté »}

11 Depuis 2005, est organisée par le gouvernement de la Nouvelle-Calédonie en partenariat avec le «Comité 150 ans après », la "fête de la citoyenneté » qui se déroule le 24 septembre sur la place nommée communément « la place du Mwâ Kââ », au centre-ville de la capitale, Nouméa. La "ville blanche » - expression communément utilisée pour parler de la ville de Nouméa dans laquelle se concentre en grande majorité la population européenne - est située en Province Sud et concentre plus d'un tiers de la population totale : 99926 habitants en 2014 pour une population totale de 268767 habitants. (La Province Sud compte 199983 habitants soit 74\% de la population totale. $)^{20}$ 


\section{Le Mwâ Kââ}

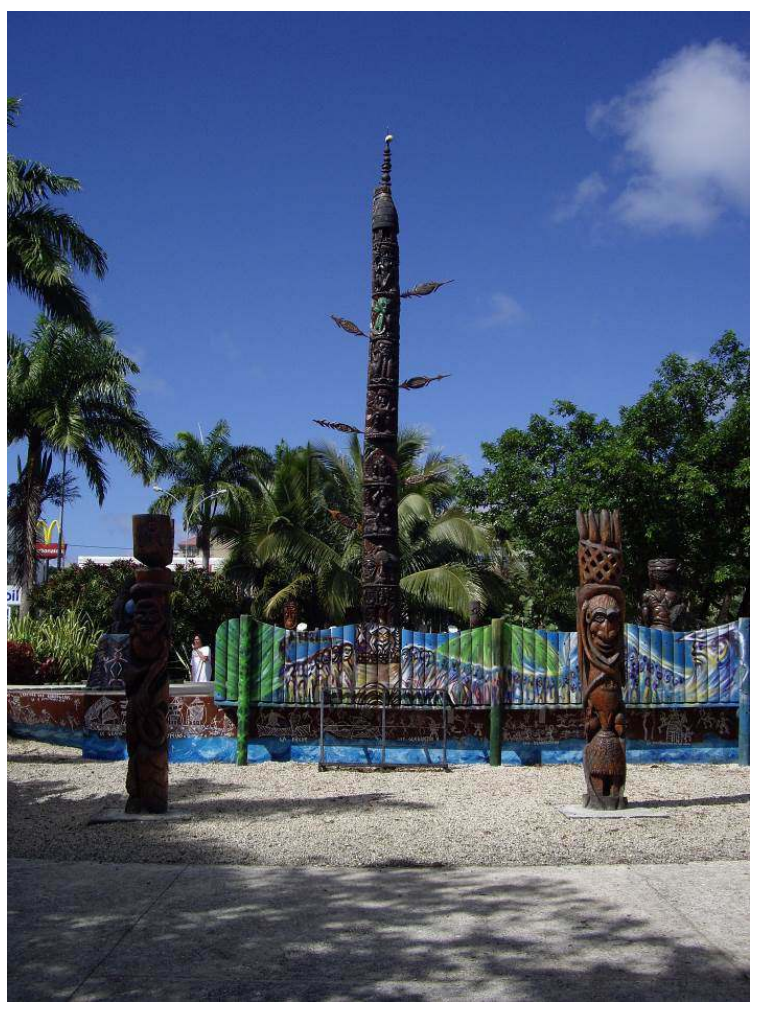

Image 2

Photo de Stéphanie Graff, 2011, Nouméa

Le 24 septembre est la date à laquelle, en 1853, l'amiral Fébvrier Despointes prit possession de la Nouvelle-Calédonie au nom de la France. Pour les indépendantistes kanak, ce jour est jour de deuil, le jour de leur colonisation ${ }^{21}$. A partir de 1872, la commémoration mit à l'honneur la France et l'armée ainsi que les bienfaits de la colonisation (Carteron 2012 : 48). La première contestation de la commémoration du 24 septembre sous cette forme a été faite en 1974 par le Groupe 1878, mouvement constitué, entre autres, de jeunes étudiants kanak revenus de France qui ont stoppé le défilé militaire en manifestant avec des banderoles, ce qui leur a valu d'être arrêtés et emprisonnés. C'est à leur sortie de prison que ces jeunes ont commencé à parler d'indépendance pour la revendiquer dans une déclaration officielle le 22 juin 1975 (Graff 2012 : 64). En 1980, une première tentative pour faire du 24 septembre la « fête de la fraternité pluriethnique » fut un échec.

En 2003, sous l'égide du Sénat coutumier ${ }^{22}$, fut créé le "Comité 150 ans après » en référence au $150^{\text {ème }}$ anniversaire de la colonisation ${ }^{23}$. Cette année-là, un mât totémique appelé Mwâ Kââ - qui peut être traduit comme « maison de l'homme de l'endroit ${ }^{24}$ » - fut érigé en face du Musée de la Nouvelle-Calédonie. Les huit sculptures sur la place et les huit flèches faittières placées sur le mât représentent les huit aires coutumières du pays kanak. Selon le "Comité 150 ans après », le Mwâ Kââ a été érigé comme rappel symbolique de la présence et de l'identité kanak dans une « ville blanche », «là où matériellement rien ne signifie la présence kanak $»^{25}$.

En 2005, année où le gouvernement de Nouvelle-Calédonie a pris en charge l'organisation de la célébration de la «fête de la citoyenneté », tout en continuant d'y associer le 
"Comité 150 ans après", des symboles reflétant l'Accord de Nouméa et un destin commun ont été ajoutés sur la place du Mwâ Kââ. Dans ce sens, un bloc de nickel a été placé à l'entrée de la place, sur lequel est gravé un passage du préambule de l'Accord de Nouméa:

Symboles du destin commun sur la place Mwâ Kââ

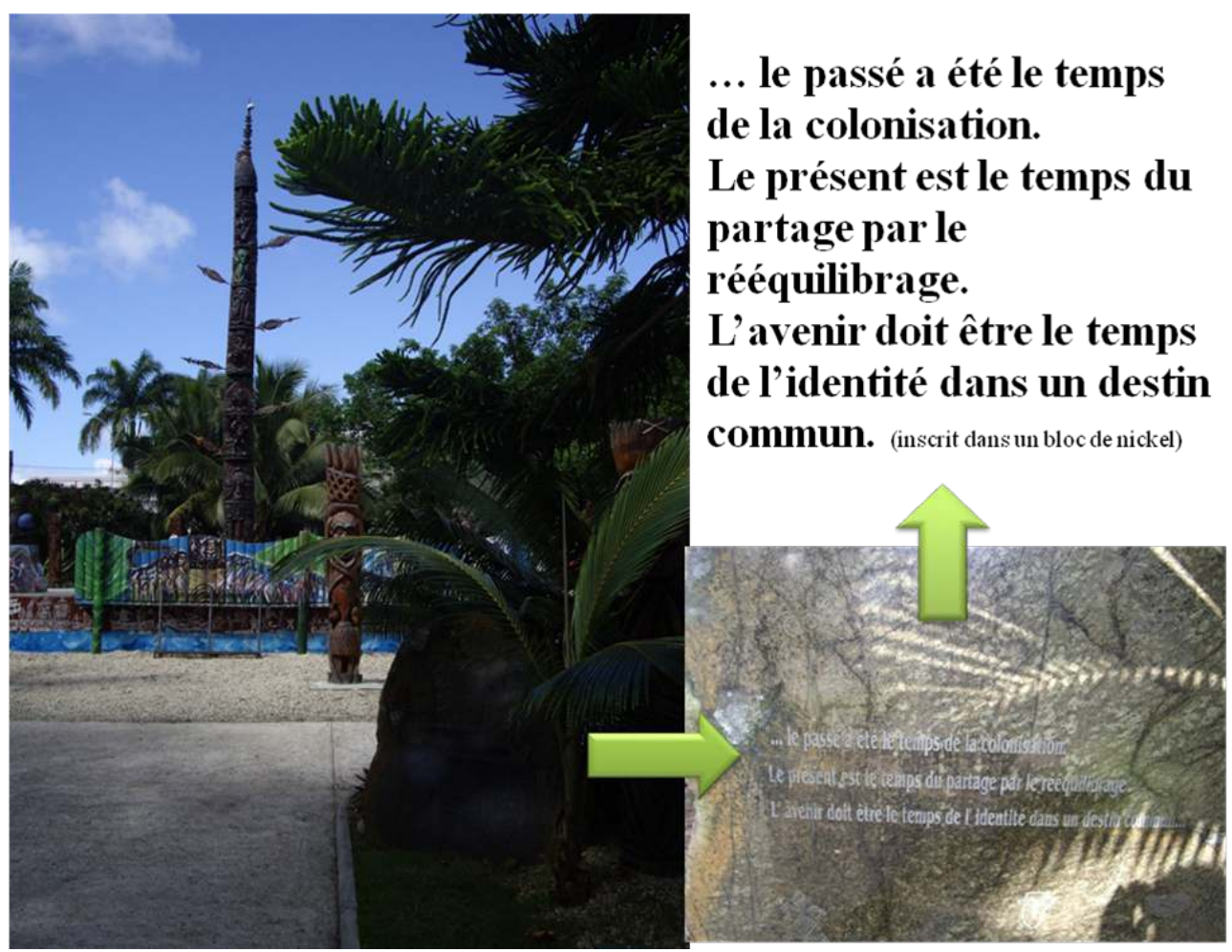

Image 3

Photo de Stéphanie Graff, 2011, Nouméa

15 Cette année-là fut également celle de l'inauguration officielle d'un nouvel aménagement de la place, redessinée afin que le mât soit implanté au milieu d'une pirogue, sur laquelle a été ajouté un "Vieux », barreur de la pirogue. Toutes les communautés ont été invitées à se rassembler sur cette « grande pirogue du destin commun » (Carteron 2012 : 53) pour la cérémonie du 24 septembre. 
Le canoë qui constitue la base du mat Mwâ Kââ, et le " Vieux », barreur du canoë

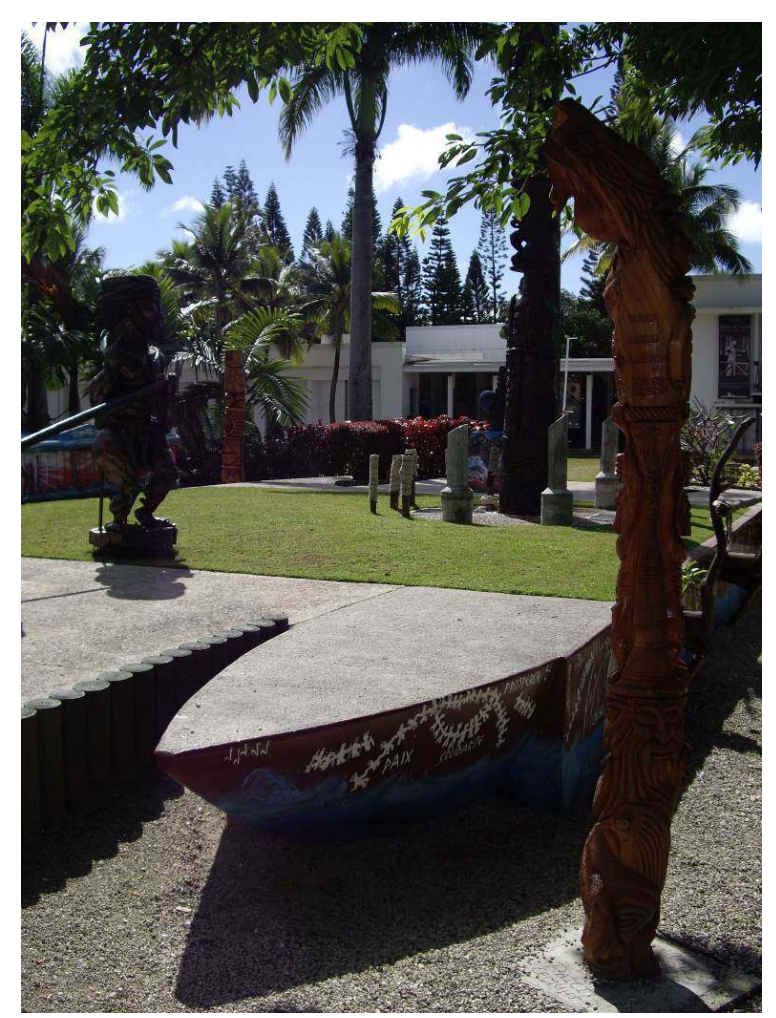

Image 4

Photo de Stéphanie Graff, 2011, Nouméa

En 2009, le gouvernement de Nouvelle-Calédonie a pris le contrôle des célébrations de la place et, l'année suivante, cette dernière fut rénovée avec des symboles supplémentaires de destin commun. Dès lors, et conformément à la " politique du destin commun ", la commémoration du Mwâ Kââ a été transformée d'une journée de deuil de colonisation en un jour de célébration de la citoyenneté calédonienne. Tous les symboles kanak, signes visuels d'une autochtonie, sont alors incorporés dans une entité calédonienne plus large. En outre, récemment, des discussions ont eu lieu afin de déterminer si la place du Mwâ Kââ devrait être rebaptisée « Place de la citoyenneté ».

Ainsi, après avoir manifesté la présence et l'identité du peuple kanak dans la ville, le Mwâ Kââ a donc été progressivement transformé en un symbole d'une identité collective calédonienne et est devenu la représentation d'un destin commun. La reconnaissance de l'identité kanak et de sa qualité de peuple autochtone devient alors simplement un préalable à la construction et à l'expression d'une identité calédonienne plus globale. Dans un article de 2012, Benoît Carteron a écrit que « le Mwâ Kââ est l'expression d'une identité offensive (Touraine, 1981) dont l'enjeu est d'opérer, à partir des référents kanak, un décloisonnement entre des groupes historiquement hostiles» (Carteron 2012: 58). Nous verrons plus loin que l'«affaire des cases ", postérieure à la publication de son article, nous permet d'en douter. 


\section{Le symbole de la poignée de main : un destin commun pour effacer le passé?}

En 2003, le "Comité 150 ans après » a produit un document accompagnant la commémoration des 150 ans de prise de possession et la pose du Mwâ Kââ.

Couverture de l'ouvrage réalisé par le « Comité 150 ans après » pour la commémoration du 150 ème anniversaire de deuil de colonisation / de la prise de possession

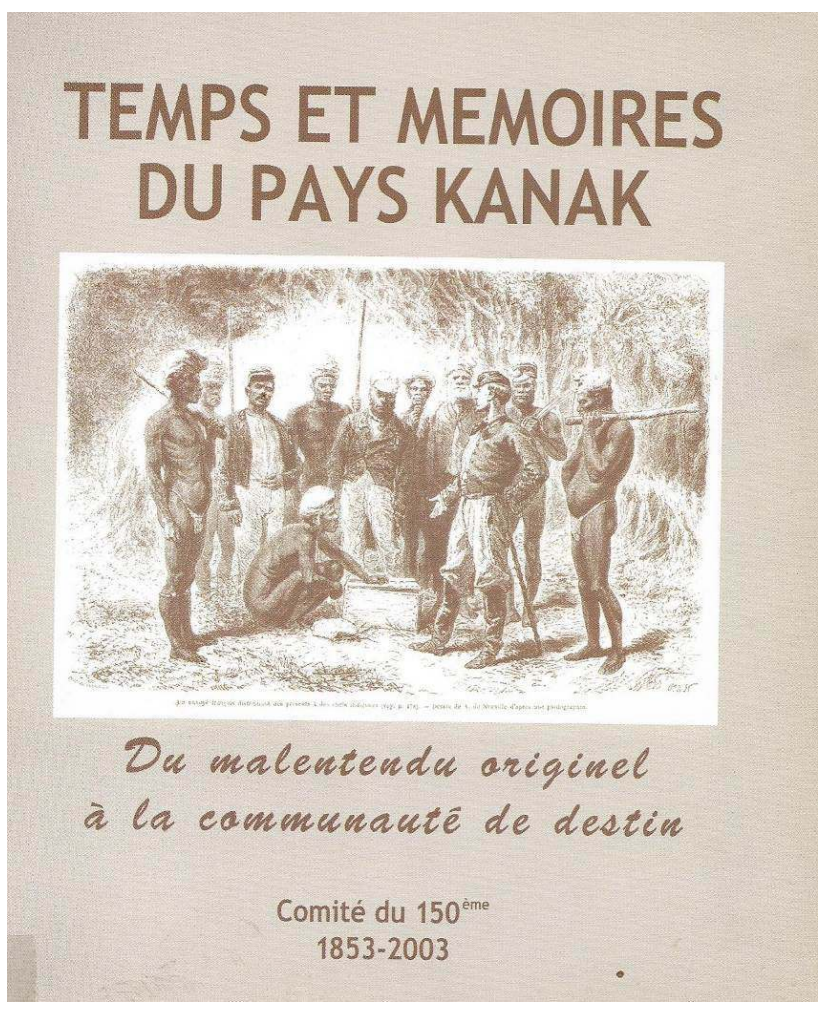

Image 5

Le titre de l'ouvrage est $\mathrm{Du}$ malentendu originel à la communauté de destin. Sur l'image accompagnant le titre on peut voir un homme "blanc», qui semble porter un uniforme de l'armée, probablement français, et un homme "noir ", probablement kanak, assis en face d'une boîte que l'homme "blanc » est en train de lui montrer. Plusieurs autres hommes «noirs ", probablement kanak, sont debout à côté et portent des vêtements militaires. La légende sur l'image indique la chose suivante: «un envoyé français distribuant des présents à des chefs indigènes ». Si l'on reprend le titre « Du malentendu originel à la communauté de destin » et qu'on regarde l'image, de quel malentendu s'agitil ? Doit-on comprendre que les représentants de l'Etat français à l'époque et les chefs kanak se seraient mal compris...? La prise de possession et la colonisation étaient-elles un « malentendu »?

Sur aucun des tableaux peints sur le canoë, ajoutés à la place du Mwâ Kââ en 2010, ne peut être trouvée une référence à la prise de possession et à la colonisation française puisque la date de 1774 est celle du débarquement de James Cook. 
21 On y raconte seulement que : «Des Vieux Kanak observent l'arrivée des Européens, le 4 septembre 1774 [donc James Cook] ».

Peinture sur la coque du canoë du Mwâ Kââ

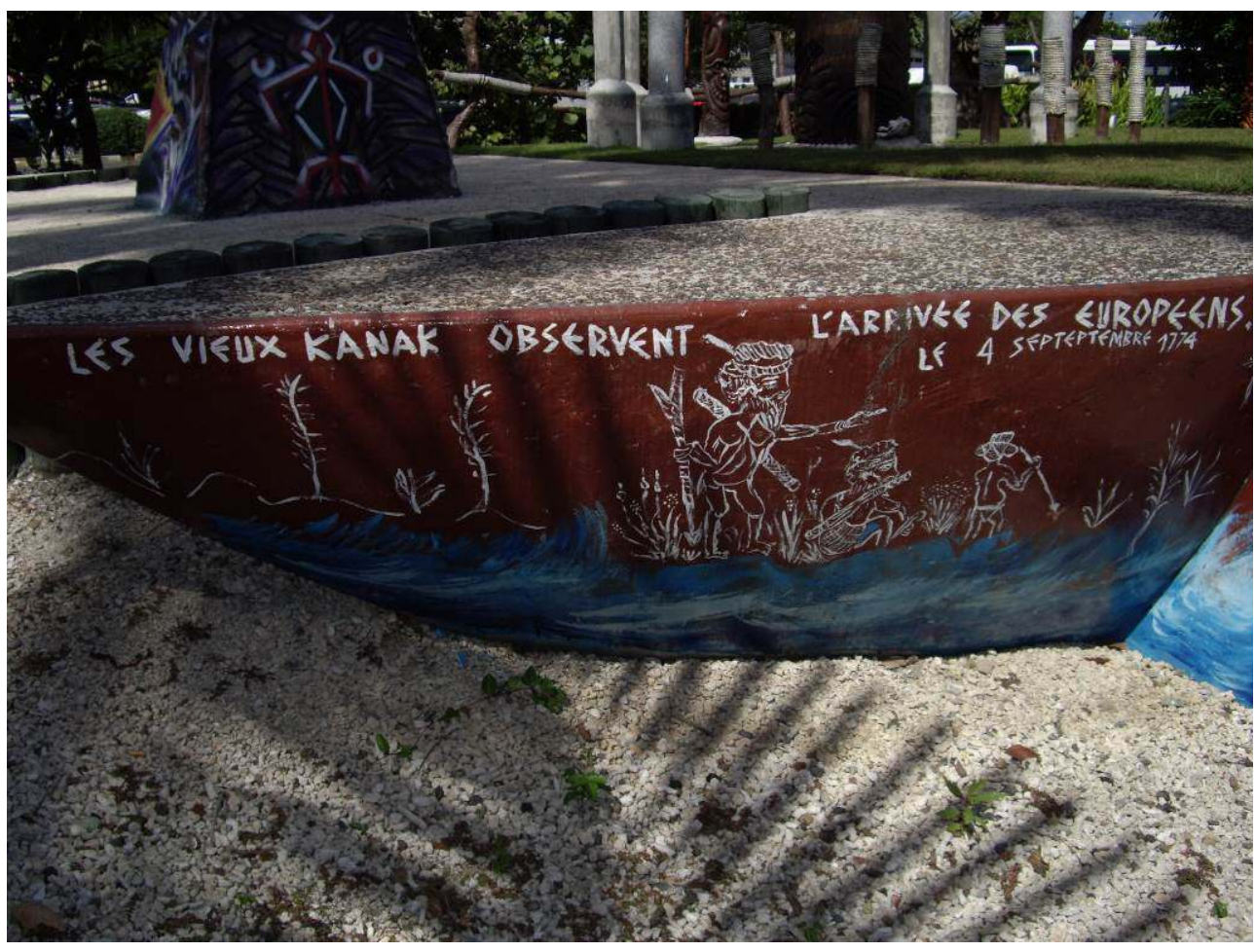

Image 6

Photo de Stéphanie Graff, 2011, Nouméa 
Peinture sur la coque du canoë du Mwâ Kââ

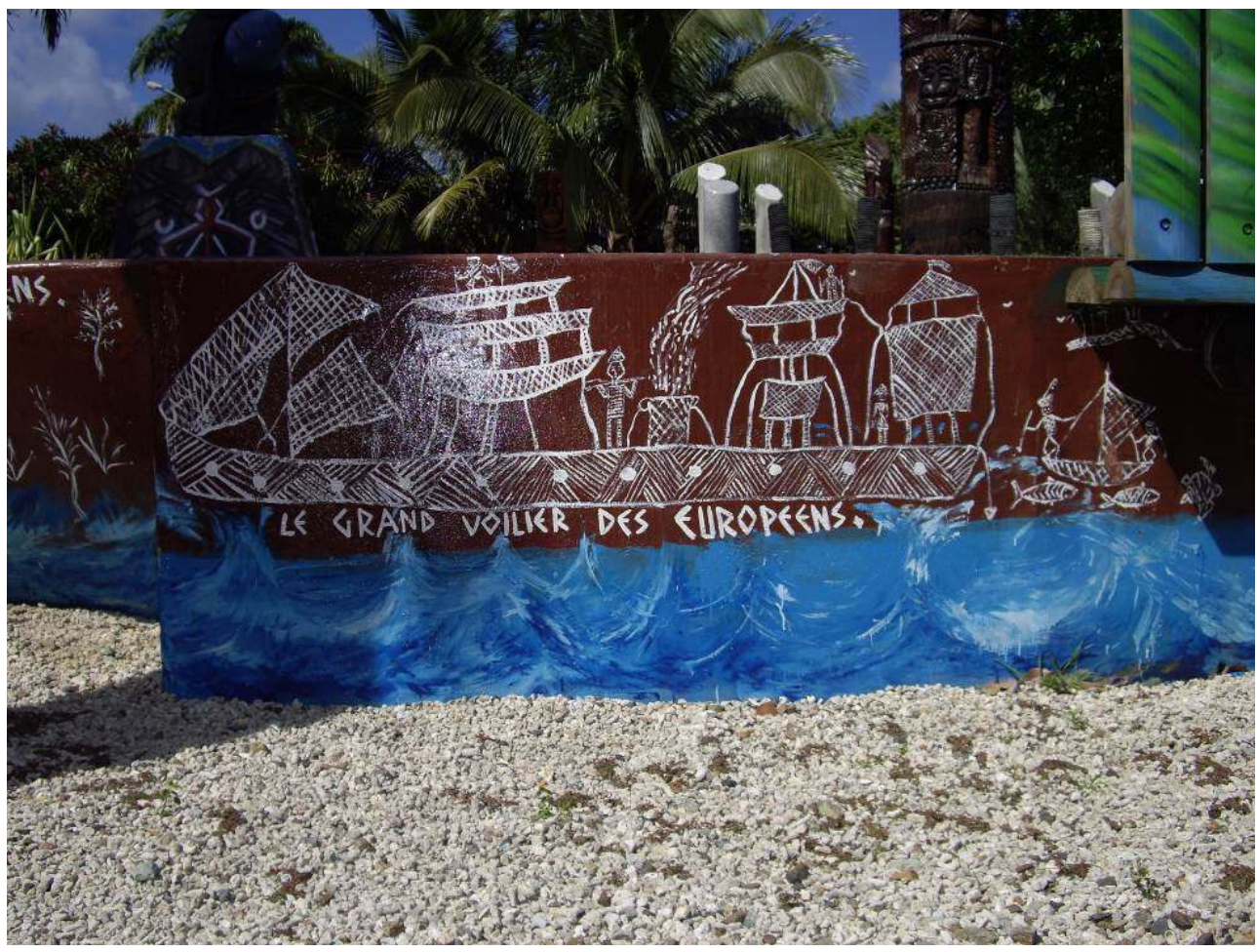

Image 7

Photo de Stéphanie Graff, 2011, Nouméa

22 Ensuite, le mode de vie traditionnel kanak (la pêche, les guerriers, les plantations, l'habitat traditionnel, et la vie en tribu) est décrit en lieu et place de l'histoire. C'est le vide historique jusqu'à... la poignée de main.

Peintures sur la coque du canoë du Mwâ Kââ
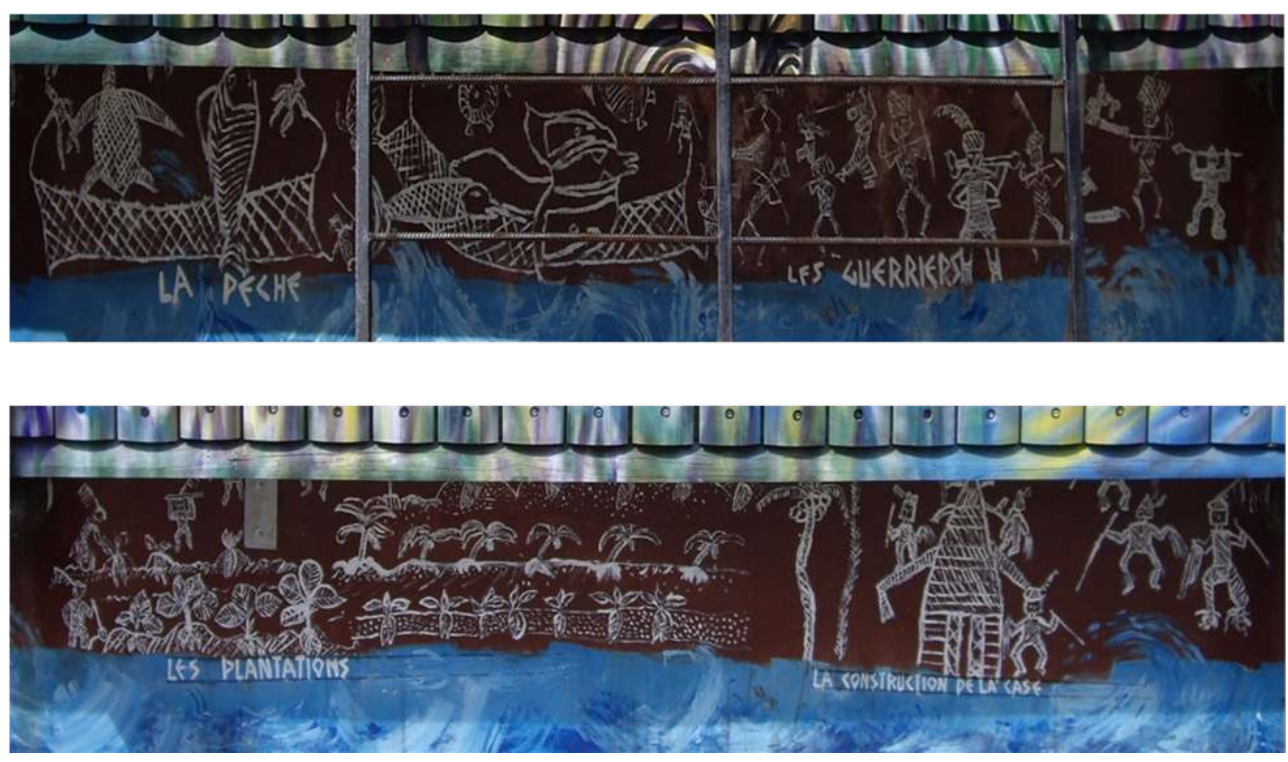

Images 8 \& 9

Photo de Stéphanie Graff, 2011, Nouméa 
Peinture sur la coque du canoë du Mwâ Kââ

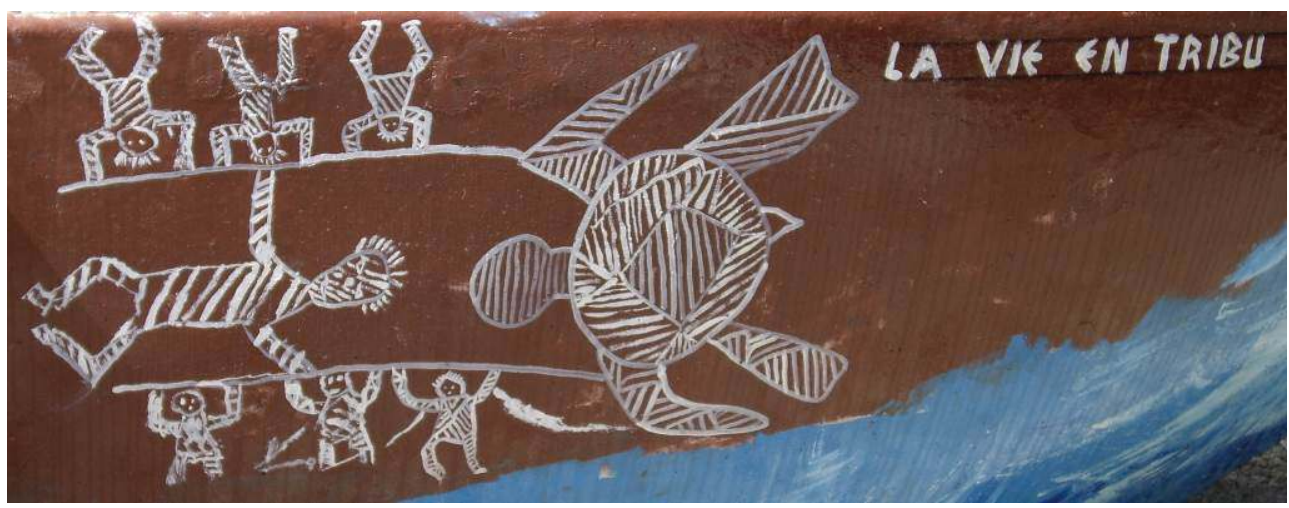

Image 10

Photo de Stéphanie Graff, 2011, Nouméa

De même, les valeurs soulignées dans l'Accord de Nouméa (paix, solidarité, prospérité, accueil) et les références aux éléments clés du développement économique (tourisme, nickel) sont mises en exergue sur les peintures du canoë.

Peinture sur la coque du canoë du Mwâ Kââ

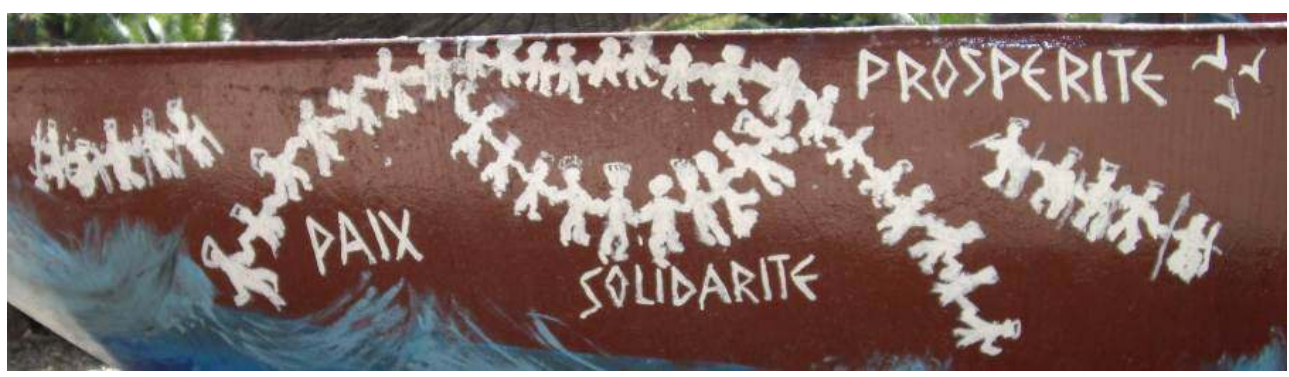

Image 11

Photo de Stéphanie Graff, 2011, Nouméa

Peinture sur la coque du canoë du Mwâ Kââ

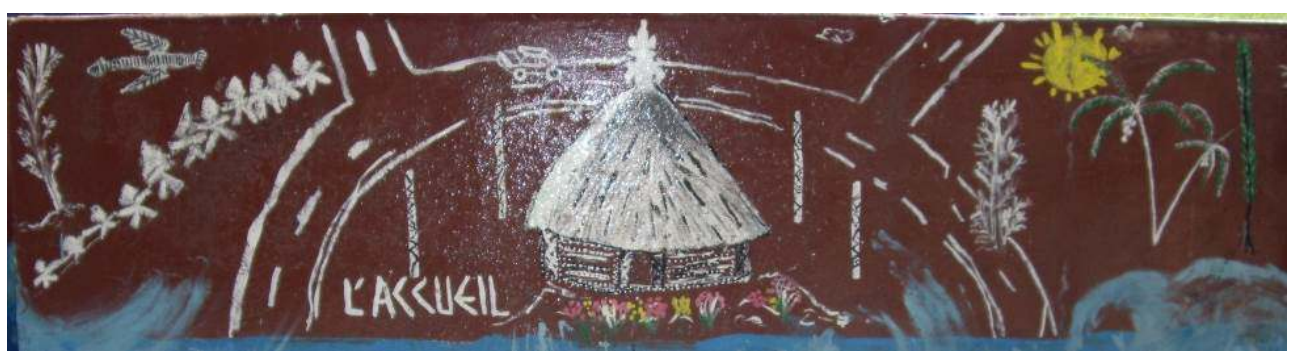

Image 12

Photo de Stéphanie Graff, 2011, Nouméa 
Peinture sur la coque du canoë du Mwâ Kââ

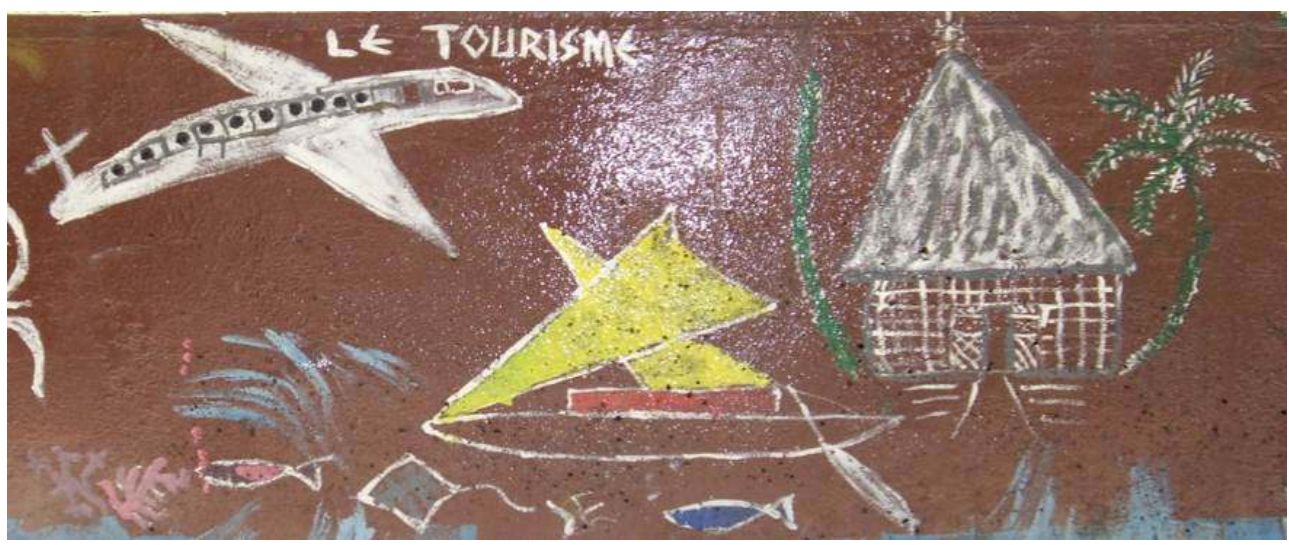

Image 13

Photo de Stéphanie Graff, 2011, Nouméa

\section{Peinture sur la coque du canoë du Mwâ Kââ}

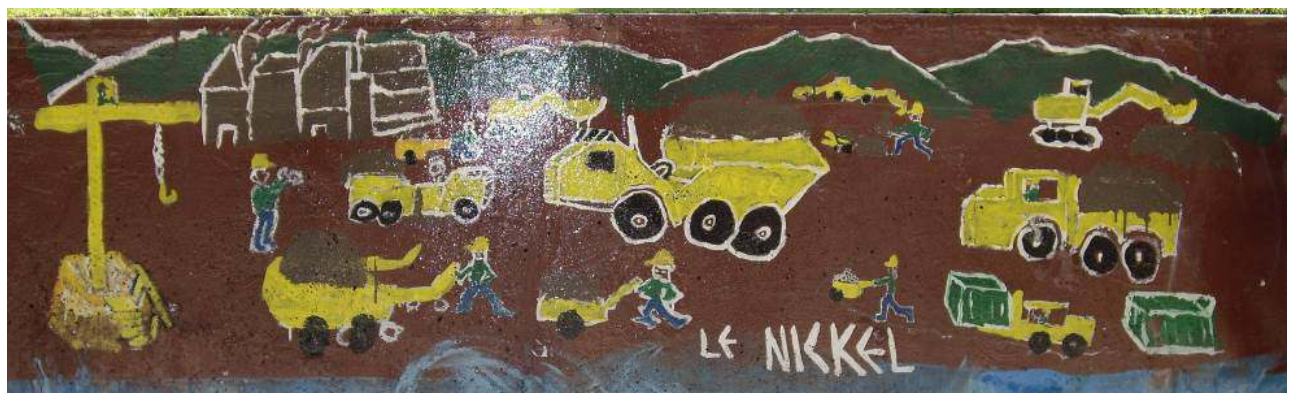

Image 14

Photo de Stéphanie Graff, 2011, Nouméa

24 Puis tout à coup apparaissent : la poignée de main, sceller un destin commun, affirmer une solidarité, se rassembler autour de l'appel du «toutoute ${ }^{26}$, sans qu'on comprenne pourquoi puisque les conflits ne sont pas évoqués.

\section{Peinture sur la coque du canoë du Mwâ Kââ}

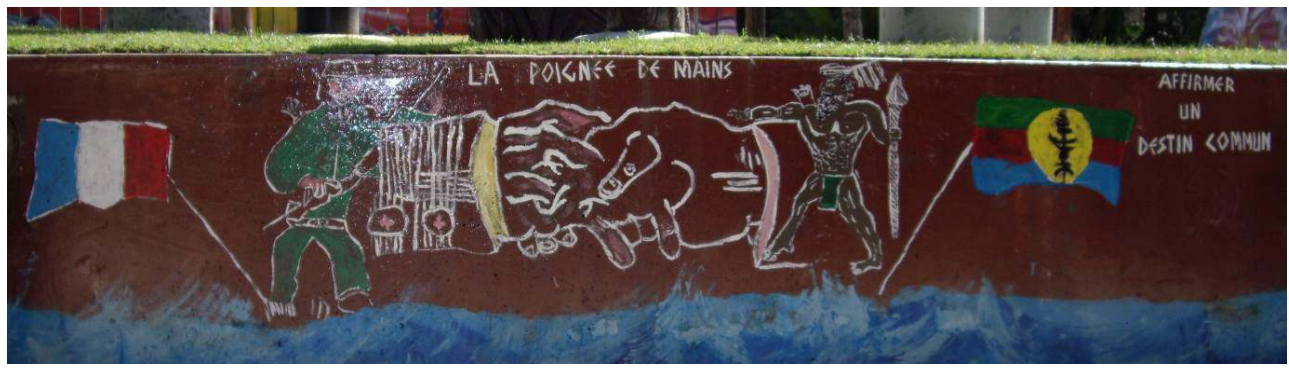

Image 15

Photo de Stéphanie Graff, 2011, Nouméa 

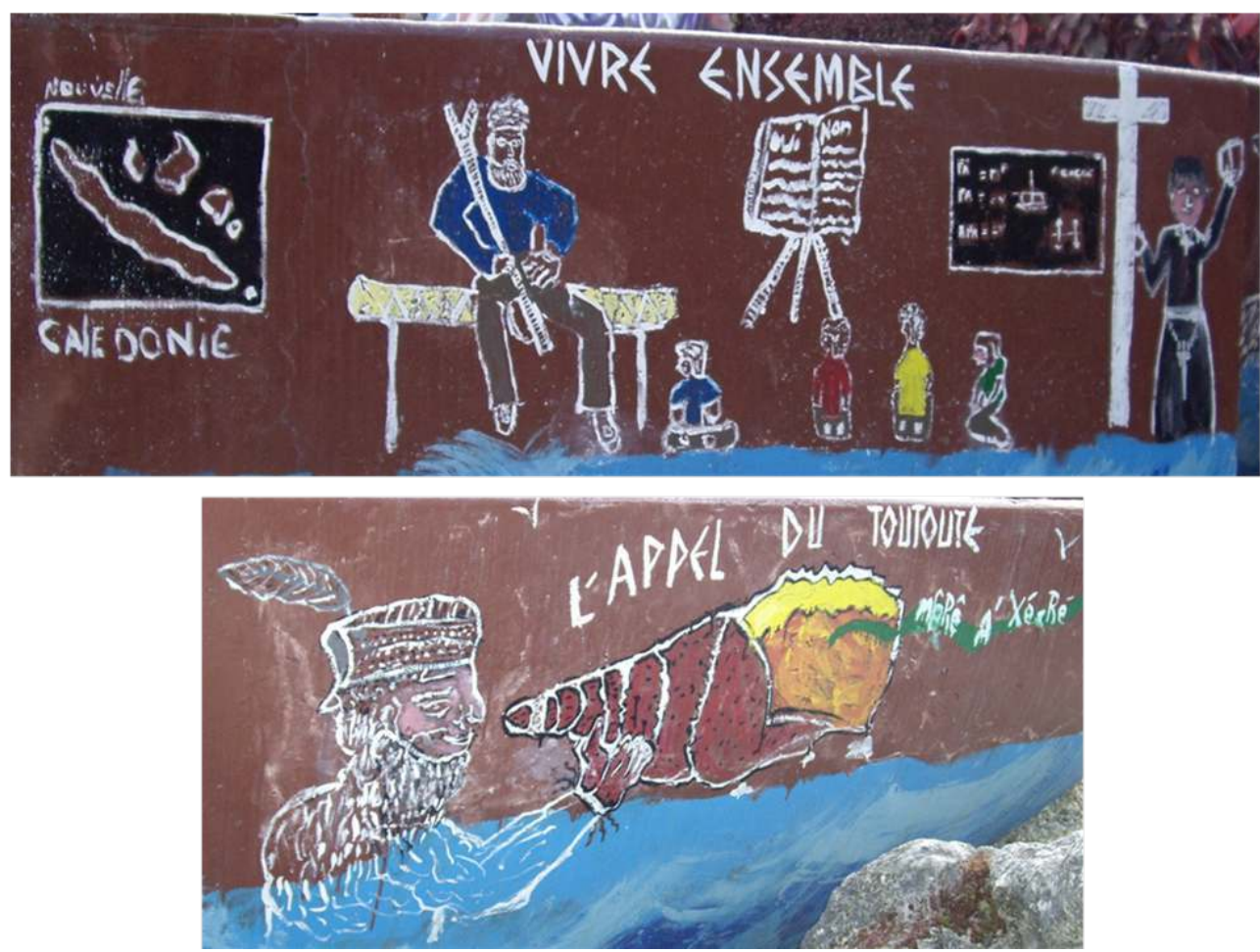

Images $16 \& 17$

Photo de Stéphanie Graff, 2011, Nouméa

Cette succession de peintures reflète l'Accord de Nouméa. Un fait colonial en parti avoué27 (images 6 \& 7), puis, comme l'écrit Geneviève Koubi (2008: 73) «la reconnaissance de l'identité kanak [images $8,9 \& 10$ ], pensée comme un préalable indispensable à la refondation du contrat social $»^{28}$ (images $11 \& 12$ ) en gardant la foi que la colonisation a amené des lumières ${ }^{29}$ (images 13 \& 14), le tout trouvant aujourd'hui sa place dans l'idée d'un destin commun (images 15, 16 \& 17). L'histoire est ainsi remaniée dans le miroir de l'Accord de Nouméa.

Lorsqu'on regarde la peinture de la poignée de main, on peut observer qu'elle se réfère à un certain type de réconciliation. En effet, la poignée de main entre Jean-Marie Tjibaou et Jacques Lafleur et celle sur la peinture du Mwâ Kââ symbolisent toutes deux la réconciliation entre les citoyens calédoniens. Dans ce registre, cette poignée de main entre le soldat «blanc » / drapeau français et le guerrier kanak / drapeau FLNKS (image 15) peut être vue comme la réconciliation entre les parties pour et contre l'indépendance. Dans cette série de poignées de main, la France n'est pas directement visible. L'acteur « Etat » apparaît seulement indirectement, derrière son soldat « blanc ».

Il convient de noter que les sculpteurs kanak, sollicités pour la réalisation du Mwâ Kââ en 2003, par le «Comité 150 ans après » à l'occasion de la commémoration des 150 ans de la prise de possession, avaient quant à eux choisi de rendre visible la colonisation, contrairement aux peintures ajoutées sur la coque en 2010. En effet, selon Benoît Carteron, « [1]a colonisation est rappelée dans les sculptures de deux aires coutumières : Hoot Ma Whaap en bas, qui a vu arriver les bateaux européens ; Djubea Kaponé, au sommet, qui a ressenti le plus vivement la colonisation avec la fondation de Nouméa sur 
ses terres. Le sculpteur de cette dernière a ainsi représenté un vieux guerrier les armes à la main pour signifier qu'il ne les a jamais déposées [...] ».

\section{$L^{\prime} \lll$ Affaires des cases $»^{30}$}

Pour la «fête de la citoyenneté » du 24 septembre 2012, sur la place du Mwâ Kââ, le "Comité 150 ans après" avait comme projet d'élaborer une tribu dans la ville en construisant neuf cases sur un parking attenant à la place du Mwâ Kââ : huit cases pour représenter chaque aire coutumière du pays kanak et la neuvième comme «case du destin commun", celle de toutes les communautés en présence sur le territoire qui ont été accueillies dans le pays kanak ${ }^{31}$.

Au mois de mai 2012, lors d'une réunion de préparation de la « fête de la citoyenneté », un accord de construction des neuf cases a été passé entre le "Comité 150 ans après », la mairie de Nouméa, le gouvernement de Nouvelle-Calédonie, et le représentant de l'Etat ${ }^{32}$. Mais peu de temps avant le 24 septembre, alors que les préparatifs étaient déjà bien avancés, la mairie retira son accord pour le projet, argumentant un problème lié aux règles de l'urbanisme. Après de nombreuses discussions avec le "Comité 150 ans après ", elle accepta à la condition que la construction des neuf cases soit provisoire et qu'elles soient retirées une fois la « fête de la citoyenneté » terminée, au plus tard le 29 septembre (Lefevre 2015: 260). Le "Comité 150 ans après " accepta. Il ne restait alors plus que quelques jours pour construire les neuf cases : la jeunesse kanak urbaine sollicitée par le « Comité 150 ans après » contribua à en construire huit.

La « Tribu dans la ville », octobre 2012

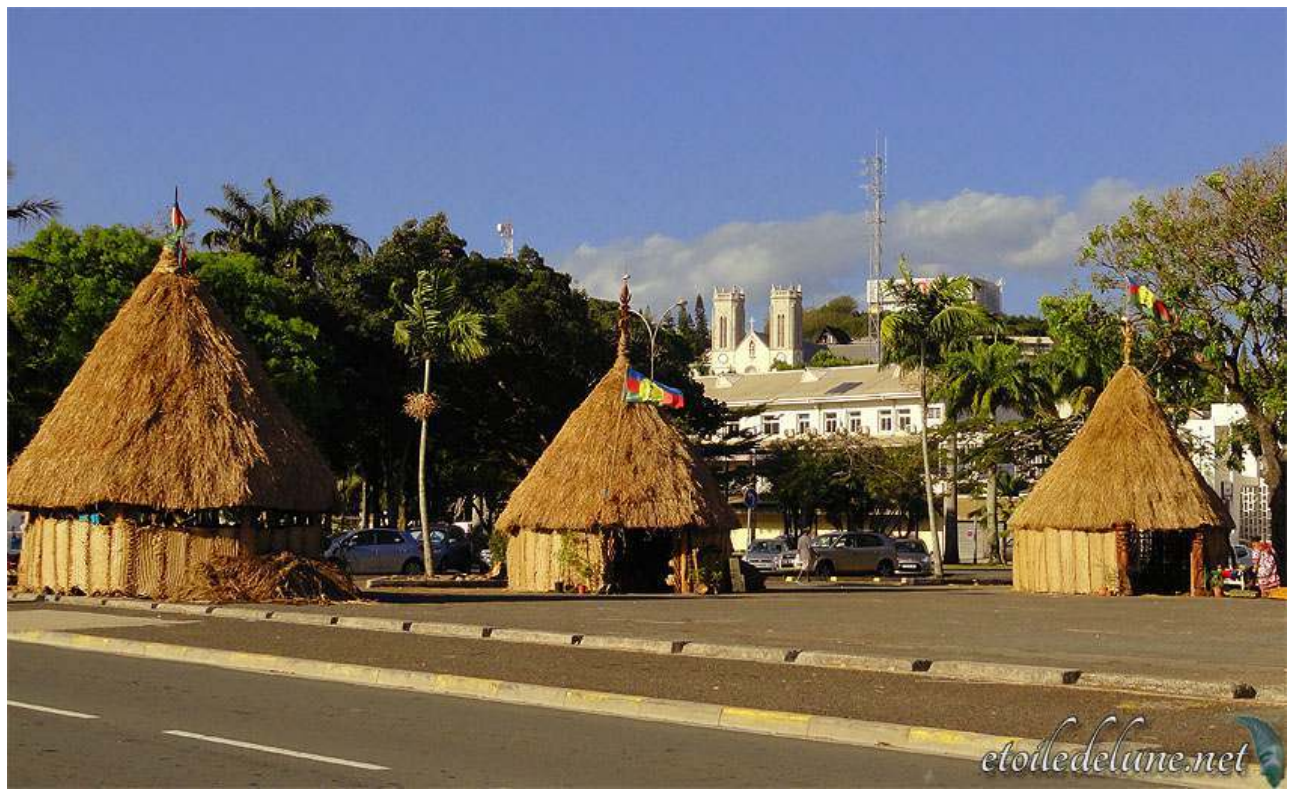

Image 18

(c) Nathalie Cathala, etoiledelune.net, http://voyage.nat-et-dom.fr/wp-content/archives/1147/image/ jpg2Wmw5f_TyV.jpg, consulté le 31/03/2016

Il y eut un malentendu autour du caractère provisoire des cases entre certains des membres du « Comité 150 ans après » et les jeunes. Beaucoup de jeunes ayant participé à 
leur construction n'ont pas compris leur caractère provisoire et/ou ne voulaient pas l'accepter. Cette divergence d'opinions sur la pérennité des cases incita les opposants à leur démolition à créer un collectif d'abord appelé « La tribu dans la ville », puis « La ville dans la tribu » qui lança une pétition pour le maintien des cases $^{33}$.

Manifestation du collectif « Tribu dans la ville »

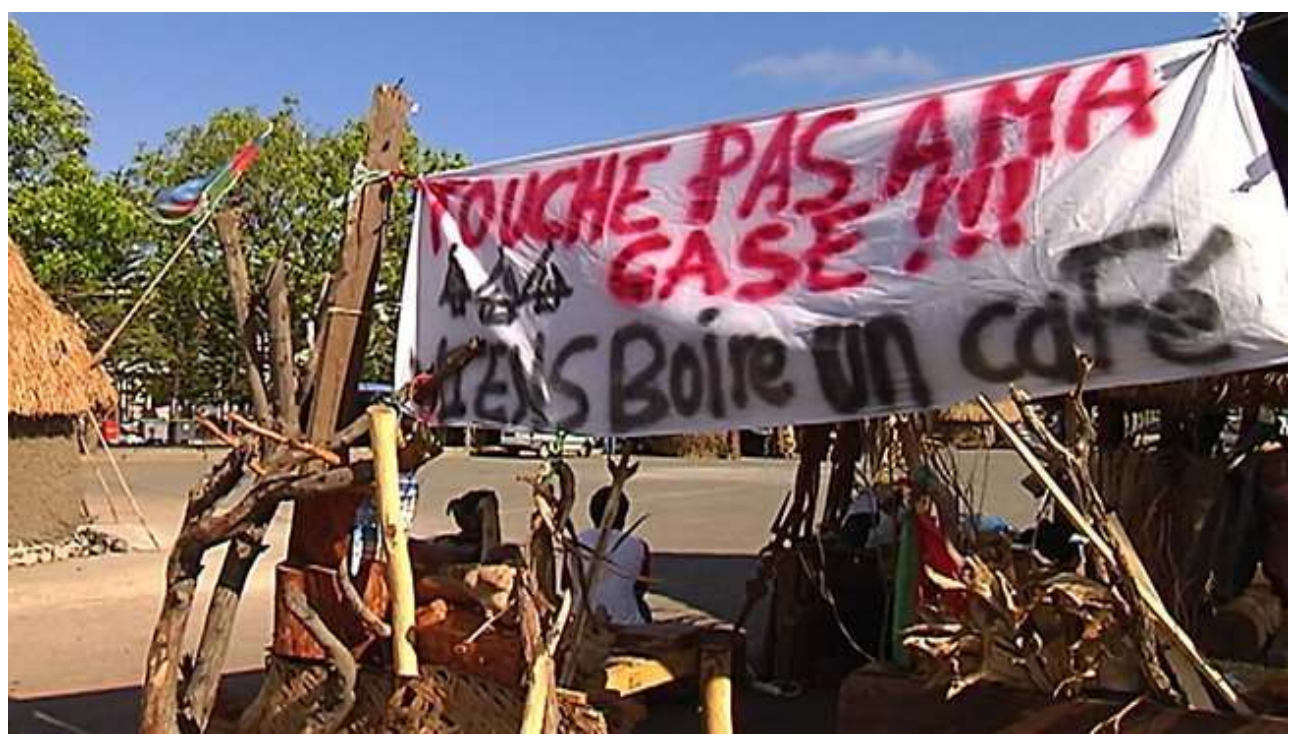

Image 20 octobre 2012

(CNC 1ère, http://la1ere.francetvinfo.fr/nouvellecaledonie/sites/regions_outremer/files/styles/top_big/ public/assets/images/retro_faitssociete.jpg?itok=H8iEZJep, consulté le 31/03/2016

31 Finalement, le 13 novembre 2012, au lever du jour, la mairie de Nouméa, sans l'autorisation légale d'un juge, fit détruire les cases par des hommes cagoulés ${ }^{34}$. Les forces de l'ordre françaises étaient présentes «en cas de troubles à l'ordre public » (Chauchat 2012). 
Destruction des cases aux bulldozers 13 novembre 2012

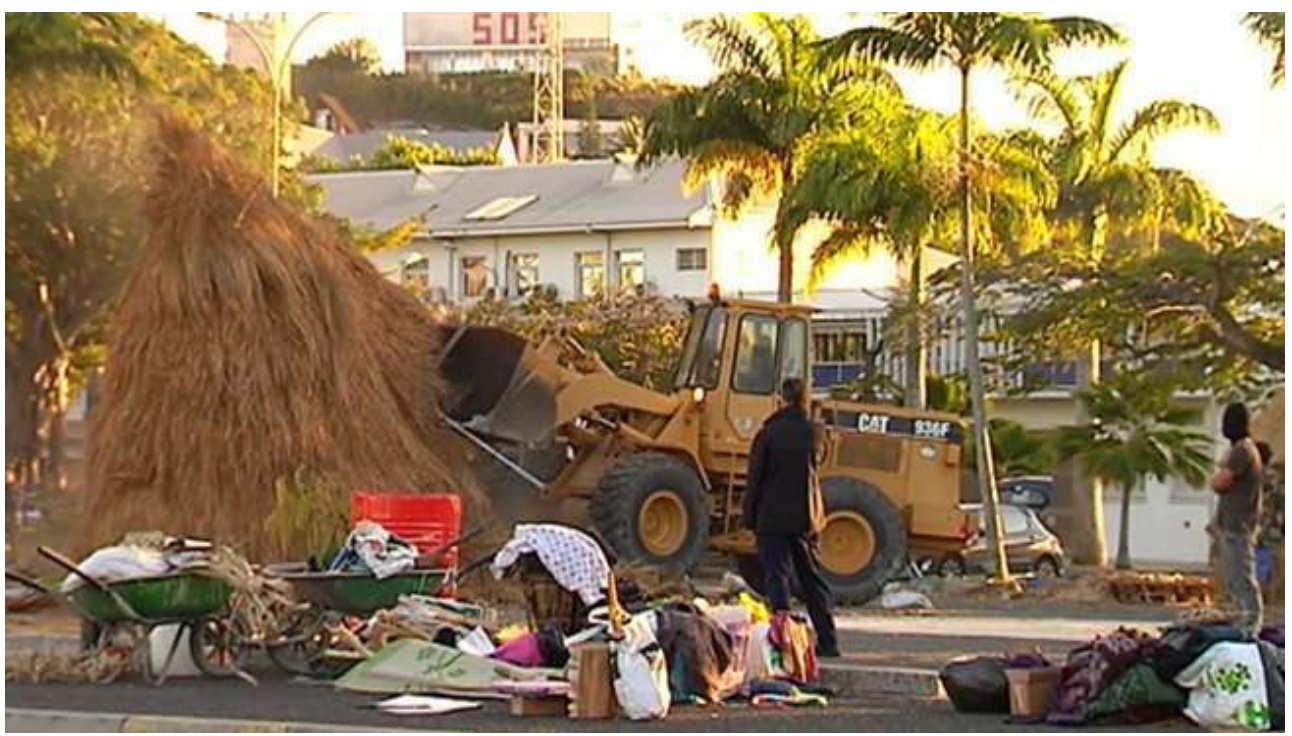

Image 21

(c) Nouvelle-Calédonie 1ère. Cédric Michaut http://la1ere.francetvinfo.fr/

nouvellecaledonie/2012/11/13/les-cases-du-mwa-ka-degagees-au-bulldozer-2164.html, consulté le $31 / 12 / 2016$

Destruction des cases aux bulldozers par des hommes cagoulés, ordonnée par la mairie de Nouméa, 13 novembre 2012

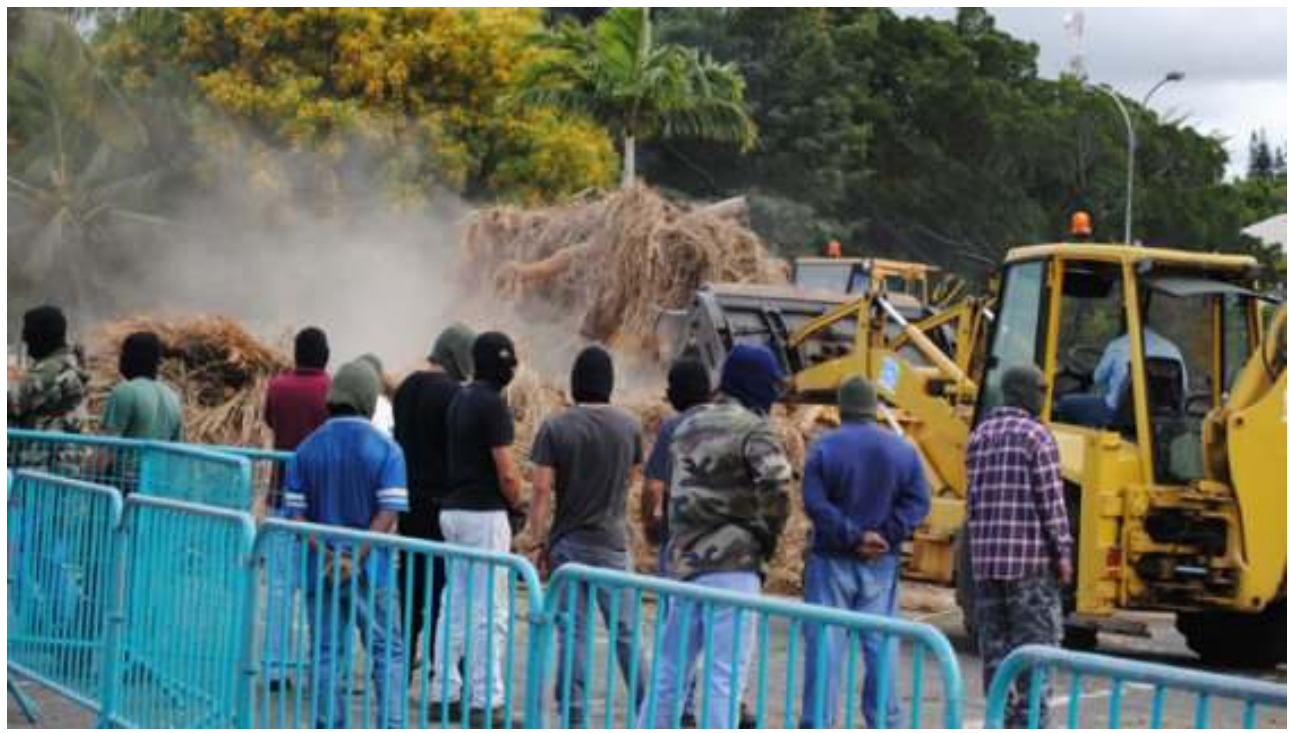

Image 22

(c) NC 1ère Daphné Gastaldi, http://la1ere.francetvinfo.fr/nouvellecaledonie/2012/11/13/les-cases-dumwa-ka-degagees-au-bulldozer-2164.html, consulté le 31/03/2016

Comme l'écrit Tate Lefevre, "[c]es évènements ont révélé jusqu’à quel point les récits coloniaux dépossèdent encore maintenant les Kanak de l'espace urbain - même si la Nouvelle-Calédonie connaît un processus de décolonisation » (2015: 255). Cette "affaire des cases" interroge sur ce que la société calédonienne dominante souhaite voir et rendre visible dans la ville. De toute évidence, il y a un rejet de voir la culture kanak au cœur de la ville, notamment comme représentant le destin commun et la citoyenneté. 
Pourtant, à la même période, les travaux d'aménagement (construction d'une porte, mise en place d'un monument commémoratif, de lampions et de sculptures) qui permettent d'identifier et de visualiser le quartier asiatique de Nouméa, étaient en cours, à une distance d'à peine un kilomètre de la place du Mwâ Kââ. N'y aurait-il que certaines communautés qui seraient bénéficiaires du destin commun?

\section{Quartier asiatique Nouméa}

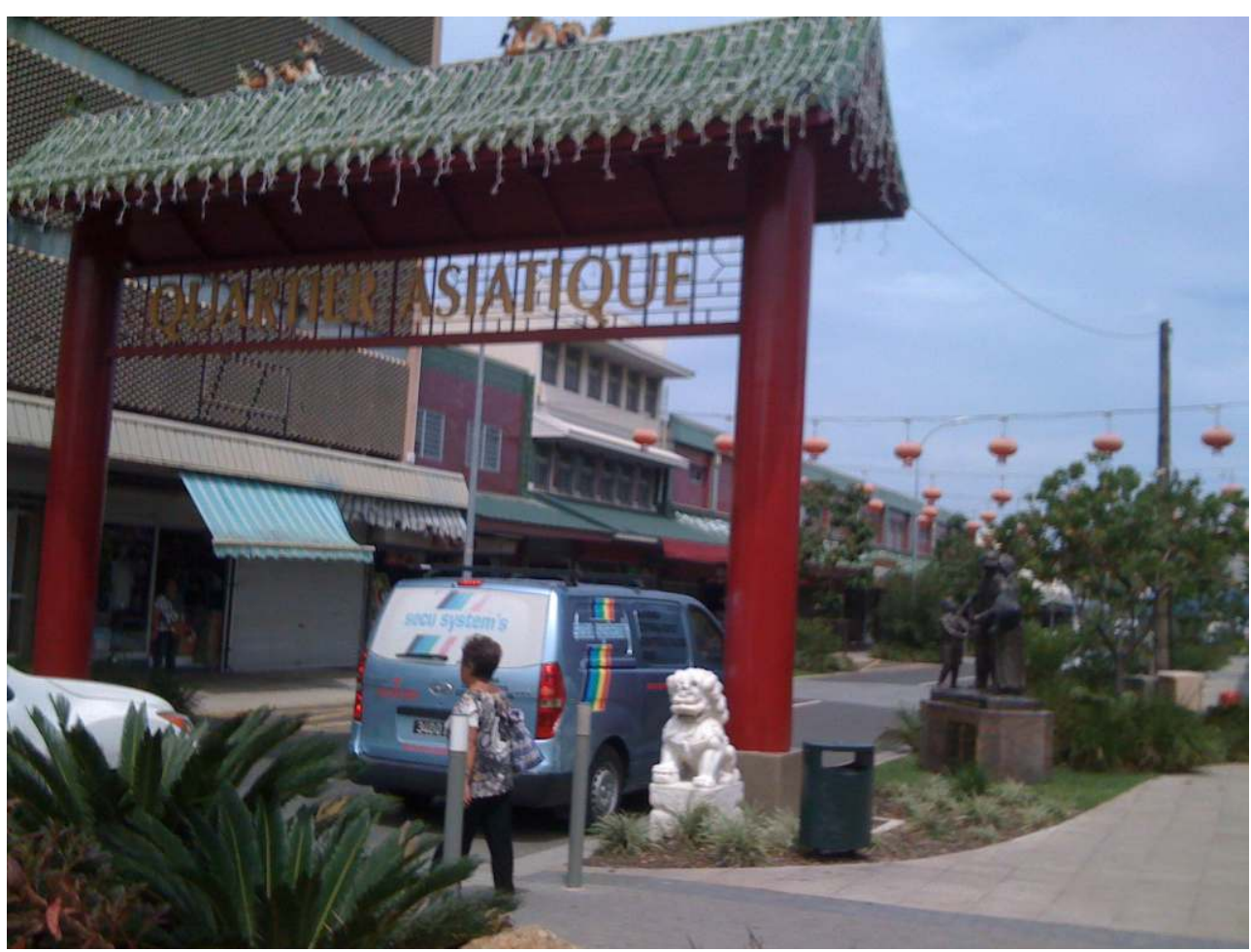

Image 23

Photo de Stéphanie Graff, 2016, Nouméa 
Vietnamiens de Nouméa posant devant le monument rendant hommage à leurs parents, les Chân Dang, lors de l'inauguration du quartier asiatique de Nouméa en octobre 2013

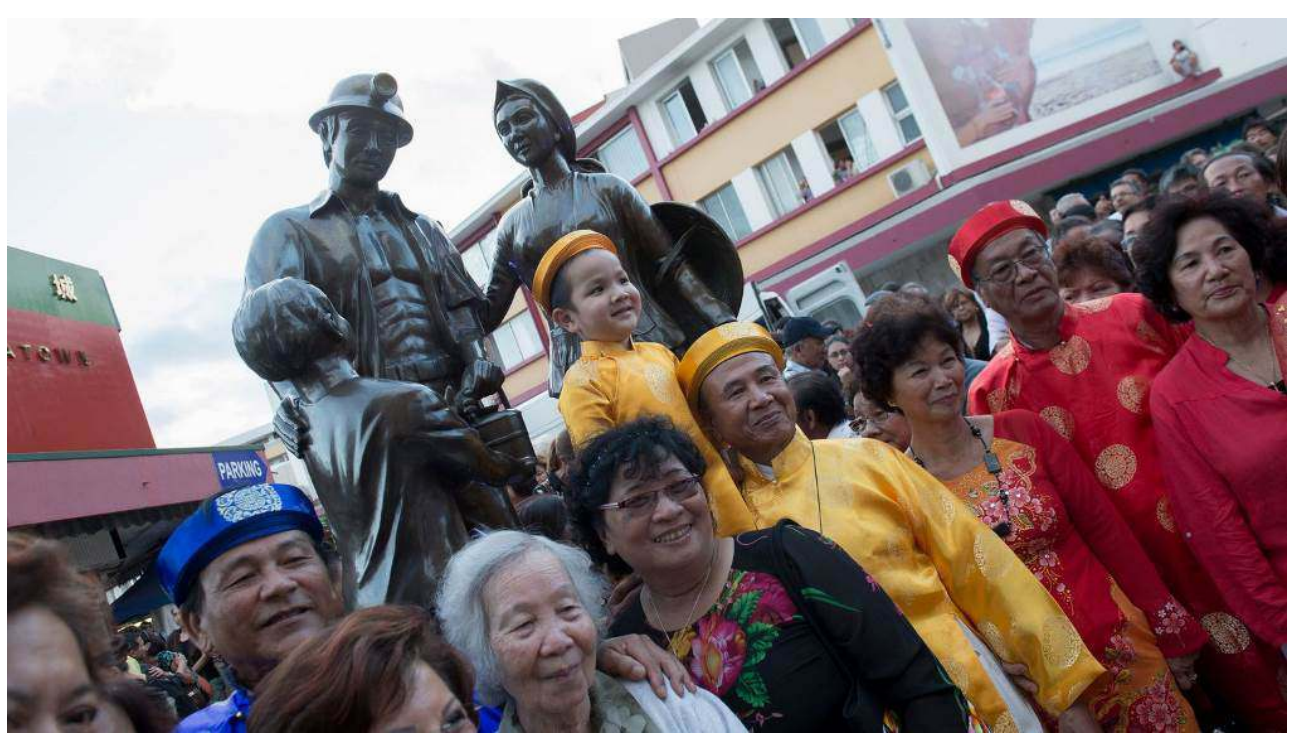

Image 24

(c) AFP/Fred Payet, http://la1ere.francetvinfo.fr/sites/regions_outremer/files/styles/top_big/public/ assets/images/2013/10/24/nc241013.jpg?itok=ckuPURtk, consulté le 31/03/2016

Cette marginalisation et cette stigmatisation de la culture kanak et des lieux de rassemblement kanak dans la ville interrogent, comme l'a soulevé Ulysse Rabaté dans sa conférence sur la jeunesse kanak (2016). Elles relèvent en effet d'une logique coloniale de contrôle social et de régulation urbaine qui nie le peuple autochtone et tente de l'acculturer dans une identité calédonienne plus globale.

Suite à «l'affaire des cases» en 2012, il n'y eut plus d'organisation commune de la commémoration du 24 septembre. En 2013, le gouvernement de la Nouvelle-Calédonie organisa la fête de la citoyenneté à Poya, tandis que le «Comité 150 ans après » organisa sa propre commémoration à Nouméa. En 2014, le gouvernement choisit Ouvéa pour célébrer la fête de la citoyenneté, alors que le FLNKS fêtait les 30 ans de sa création, le 24 septembre, à Nouméa. En 2015, le gouvernement organisa la fête du 24 septembre à la tribu de N'Dé, pendant que le "Comité 150 ans après » s'associait à la célébration des 30 ans de Radio Djiido ${ }^{35}$, le même jour, sur la place du Mwâ Kââ.

\section{Conclusion : limites et répercussions de la « politique du destin commun »}

L'« affaire des cases » fut traumatisante pour l'ensemble des acteurs kanak qui l'ont vécue et a ravivé les tensions autour de la cérémonie du 24 septembre. La vision de la destruction des cases au bulldozer est restée gravée dans les esprits, car il s'agissait avant tout de revendiquer un "droit à la visibilité ${ }^{36}$ » au cœur de la "ville blanche ». L'analyse que fait Tate Lefevre dans son article soulève ce point en mettant l'accent sur la revendication identitaire du peuple kanak : «[...] il s'agit d'une revendication identitaire liée au «droit à la ville blanche », la "tribu dans la ville» doit avant tout être comprise comme une demande insistante de reconnaissance de la légitimité de l'identité kanak [... ]» (2015 : 255). Néanmoins le refus d'une partie des acteurs kanak, notamment les jeunes, 
d'accepter le caractère provisoire des cases, imposé finalement par la mairie, peut être perçu également comme une stratégie de résistance. Celle-ci est non seulement culturelle, vis-à-vis de pouvoirs publics qui tentent d'effacer les expressions visuelles et visibles d'une culture kanak spontanée dans l'espace urbain, mais aussi une stratégie de résistance politique et d'affirmation d'une revendication historique d'indépendance et de regain de souveraineté. Car une grande partie du peuple kanak, surtout les jeunes, est laissée à la marge d'un système culturellement et politiquement assimilant. C'est ainsi que s'opère également, au sein du peuple kanak, une division entre ceux qui ont choisi de monter dans le wagon des institutions, ceux qui s'y refusent, et ceux qui sont laissés sur le bord de la route.

En conclusion, les tentatives d'attiser la reconnaissance d'un destin commun entre les personnes et les communautés, de créer une identité collective calédonienne partagée existent, mais chaque personne interprète avec sa propre représentation ce qu'est, ou devrait être, le destin commun. Pour certains le destin commun et la citoyenneté calédonienne ne sont que mensonge ou illusion au regard des discriminations et inégalités qui subsistent en Nouvelle Calédonie. Les disparités sociales et économiques entre les populations autochtones et non autochtones sont en effet difficiles à ignorer.

Photo de gauche: Manifestation du collectif pour un drapeau commun, 25 août 2011 Photo de droite : Graffiti sur la Save-Express (voie rapide) de Nouméa, mars 2012

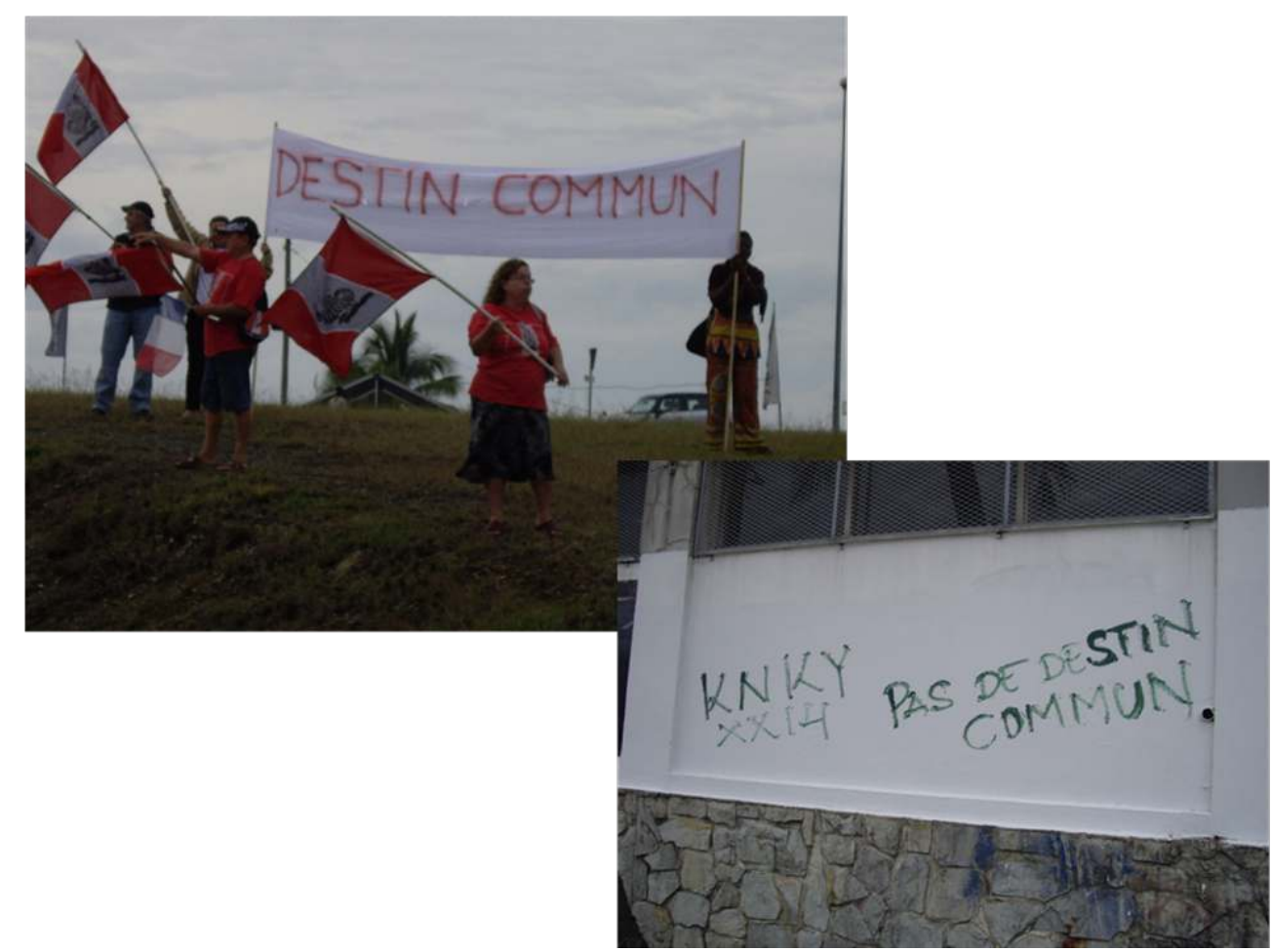

Images $25 \& 26$

Photo de Stéphanie Graff, 2016, Nouméa

Malgré la tentative de faire disparaître dans une citoyenneté calédonienne et un destin commun les deux blocs indépendantiste et non-indépendantiste ayant depuis fort longtemps une vision opposée de l'avenir, les tensions et polémiques autour du Mwâ Kââ prouvent que si celles-ci sont de l'ordre du non-dit, elles n'en sont pas moins réelles. Ainsi, un membre du « Comité 150 ans après » a souligné que le 24 septembre étant lié à la 
prise de possession, il est impossible de dissocier cette date de son histoire, même si certains pensent qu'il faut faire table rase du passé pour laisser place à une citoyenneté calédonienne et un destin commun.

La version officielle de l'histoire donnée en Nouvelle-Calédonie fabrique des images, des symboles et des mythes pour créer l'histoire présente et future, mais aussi le souvenir collectif de l'histoire passée. Selon Linda Tuhiwai Smith: « [h]istory is important for understanding the present and that reclaiming history is a critical and essential aspect of decolonization » (1999: 29-30). Il est donc important pour le peuple kanak, comme pour les autres peuples autochtones, d'écrire leur propre histoire.

Les conflits qui historiquement ont secoué la Nouvelle-Calédonie, et sont toujours d'actualité, portaient non pas sur le fait de vouloir ou non un destin commun, mais bien sur la question de l'indépendance.

40 La version historique officielle des conflits en Nouvelle-Calédonie sous-entend qu'il s'agissait de conflits ethniques entre le peuple kanak et les communautés non-kanak. Or, s'il existe un conflit ethnique, il résulte de l'instrumentalisation politique des populations hétéroclites en présence qui dissimule le fait que le fond du problème n'est pas l'ethnie, mais bien le fait colonial toujours actuel. Il y a toujours eu des individus non-kanak soutenant l'indépendance du peuple kanak et de la Nouvelle-Calédonie, comme des individus kanak étant contre l'indépendance. Le conflit est d'abord politique car entre partisans de l'indépendance et partisans du maintien de la Nouvelle-Calédonie dans la France. Les communautés non-kanak étant en grande majorité contre l'indépendance, l'amalgame est très rapidement fait. Etant issu de la prise de possession par la France, ce conflit sur la souveraineté reste un conflit colonial. La réécriture de l'histoire qui vise à faire croire que le conflit est ethnique et non politique offre à la France l'avantage de pouvoir se positionner en tant qu'arbitre et garant de la paix sociale.

41 Aujourd'hui il est très fréquemment sous-entendu que vouloir l'indépendance c'est être contre le destin commun. Or, le destin commun ne peut-il pas être envisagé dans un pays indépendant?

42 La finalité de la " politique du destin commun » est peut-être de masquer cette question brûlante de l'accession du territoire à la pleine souveraineté, la faire oublier en donnant un maximum d'autonomie et en fabriquant une citoyenneté calédonienne mais qui doit tout de même rester un sous-ensemble de la nationalité française. Cette stratégie politique tente de présenter la société calédonienne comme une société multicommunautaire noyant dans un tout le peuple autochtone et colonisé, qui historiquement revendique son indépendance.

43 "L'affaire des cases» montre que le chemin du destin commun et de la réconciliation entre le peuple et les communautés de Nouvelle-Calédonie est encore long, et que la négation et la marginalisation du peuple autochtone et colonisé est toujours d'actualité. Le destin commun semble être un chemin à sens unique sous-entendant l'assimilation et l'acculturation des Kanak à une société qui se veut calédonienne. C'est ainsi que dans son discours d'investiture en 2015, le président du Congrès Thierry Santa prononçait ces mots : «Je vous avais [...] fait part du rêve qui m'anime. Le rêve qu'un jour nous ne parlerons plus du peuple premier, ni des victimes de l'histoire, mais uniquement $\mathrm{du}$ peuple calédonien. » 


\section{BIBLIOGRAPHIE}

Accords de Matignon-Oudinot, 26 juin et 20 août 1988.

Accord de Nouméa, 5 mai 1998.

Albert, Bruce. 1997. Situation ethnographique et mouvements ethniques. Réflexions sur le terrain post-malinowskien, In Anthropologues en danger. Agier M. (éd.). Pp.75-88. Ed. Jean-Michel Place.

Anaya, James. 2000. Indigenous Peoples in International Law. New York, Oxford: Oxford University Press.

Asch, Michael et al. 2004. Discussion: On the Return of the Native. Current Anthropology 45(2): 262-267.

Asch, Michael et al. 2006. More on the Return of the Native. Current Anthropology 47(1): 145-149.

Baissat, Bernard, dir. 2006. La grande tournée outre-mer du Général De Gaulle. Mano a Mano, RFO (production). $52 \mathrm{~min}$.

Barnard, Alan. 2004. A response to Justin Kenrick and Jerome Lewis. Anthropology Today 20(5): 19.

Barnard, Alan. 2006. Kalahari revisionism, Vienna, and the "indigenous peoples" debate. Social Anthropology 14(1): 1-16.

Bellier, Irène, dir. 2013. Peuples autochtones dans le monde : les enjeux de la reconnaissance, Paris : L'Harmattan.

Bertrand, Romain. 2006. Mémoires d'empire. La controverse autour du « fait colonial ». Éditions du Croquant.

Bosa, Bastien. 2008. À l'épreuve des comités d'éthique. Des codes aux pratiques. In Les politiques de l'enquête. Epreuves ethnographiques. Didier Fassin et Alban Bensa, dir. Pp.205-225. Paris: Ed. La Découverte.

Bowen, John. 2000. Should we have a universal concept of "indigenous peoples' rights": ethnicity and essentialism in the twenty-first century". Anthropology Today 16(4): 12-16.

Carteron, Benoît. 2012. Le Mwâ Kââ, vers la manifestation d'une appartenance commune ? Journal de la Société des Océanistes 134(2012-1): 45-60.

Cefaï, Daniel, dir. 2010. L'engagement ethnographique. Paris : Editions de l'Ecole des Hautes Etudes en Sciences Sociales.

Chauchat, Mathias. 2012. La brutalité du gel du corps électoral. http://larje.univ-nc.nc/ index.php/les-travaux/faits-et-analyses/273-la-brutalite-du-gel-du-corps-electoral, (consulté le $31 / 12 / 2016)$.

Chauchat, Mathias. 2012. La destruction illégale des cases kanak. http://larje.univ-nc.nc/ index.php/les-travaux/faits-et-analyses/400-la-destruction-illegale-des-cases-kanak, (consulté le 31/12/2016).

Clinchamps, Nicolas. 2008. Distorsions et corps électoraux en Nouvelle-Calédonie. Pouvoirs 127 : 151-165. 
Daes, Erica-Irene A. 1986. Native People's Rights. Les Cahiers de droit 27(1): 123-133.

Daes, Erica-Irene A. 1993. Some Considerations on the Right of Indigenous Peoples to SelfDetermination. Transnational Law and Contemporary Problems 3(1): 35-45.

Faberon, Jean-Yves. 2001. L'évolution du droit de vote en Nouvelle-Calédonie. Revue juridique polynésienne 1(Hors-Série).

Gabriel, Claude et Vincent Kermel. 1985. Nouvelle-Calédonie. La révolte kanake. Paris : La Brèche. Giabiconi, Julie. 2012. Femmes européennes, terrains amérindiens : terra nullius de l'éthique? Ethique publique 14(2012-1) https://ethiquepublique.revues.org/954, (consulté le 31/12/2016).

Graff, Stéphanie. 2012. Quand combat et revendications kanak ou politique de l'Etat français manient indépendance, décolonisation, autodétermination et autochtonie en Nouvelle-Calédonie. Journal de la Société des Océanistes 134(2012-1) : 61-83.

Graff, Stéphanie. 2015. Autodétermination et autochtonie en Nouvelle-Calédonie. L'effacement progressif de la question coloniale. Thèse de doctorat en Etudes du développement, Institut des Hautes Etudes Internationales et du Développement, Genève.

Hastrup, Kirsten et al. 1990. Anthropological Advocacy: A Contradiction in Terms? Current Anthropology 31(3): 301-311.

Kenrick, Justin and Lewis Jerome. 2004. Indigenous peoples' rights and the politics of the term “indigenous. Anthropology Today 20(2): 4-9.

Koubi, Geneviève. 2008. Sur les méfaits de la colonisation. Cités 36(4): 63-76, P.U.F.

Koubi, Geneviève et Isabelle Schulte-Tenckhoff. 2000. "Peuple autochtone" et "minorité" dans les discours juridiques : imbrications et dissociations. Revue interdisciplinaire d'études juridiques 45: $1-26$.

Kuper, Adam. 2003. The Return of the Native. Current Anthropology 44(3): 389-402.

Lam, Maivan Clech. 2000. At the Edge of the State: Indigenous Peoples and Self-Determination. Ardsley New York: Transnational Publishers.

Leblic, Isabelle. 1993. Les Kanak face au développement. La voie étroite. Grenoble: Presses universitaires de Grenoble/ Agence de développement de la culture kanak.

Lefevre, Tate. 2015. « Nous ne sommes pas des délinquants! ». L'autorité coutumière et la marginalisation de la jeunesse urbaine kanak. Ethnies 37-38: 254-267.

Makaremi, Chowra. 2008. Participer en observant. Etudier et assister les étrangers aux frontières. In Les politiques de l'enquête. Epreuves ethnographiques. Didier Fassin et Alban Bensa, dir. Pp. 165183. Paris: Ed. La Découverte.

Marcus, George E. 1995. Ethnography In/Of the World System: The Emergence of Multi-Sited Ethnography. Annual Review of Anthropology 24: 95-117.

Merle, Isabelle. 1995. Expériences coloniales. La Nouvelle-Calédonie (1853-1920). Paris: Belin.

Merle, Isabelle. 2004. De la « légalisation » de la violence en contexte colonial. Le régime de l'indigénat en question. Politix 17(66): 137-162.

Mohamed-Gaillard, Sarah. 2010. Les élections législatives en 1951 en Nouvelle-Calédonie et dépendances. In Les élections législatives et sénatoriales Outre-mer. Laurent Jalabert, Bertrand Joly, Jacques Weber, dir. Pp. 477-486. Paris: Les Indes Savantes. 
Monnerie, Denis. 2005. La Parole de notre Maison, Discours et cérémonies kanak aujourd'hui (NouvelleCalédonie). Paris : CNRS Éditions et Éditions de la maison des sciences de l'homme.

Pantz, Pierre-Christophe. 2015. Géopolitique des territoires kanak. Décolonisation et reconquête plurielle des territoires. Thèse de doctorat en géographie, Université Paris 1.

Rabaté, Ulysse. 2016. Quand la jeunesse calédonienne prendra la parole. Séminaire interinstitutionnel de recherche en sciences sociales, Institut de Recherche pour le Développement, Nouméa, 2 mars 2016.

Scheper-Hugues, Nancy. 1995. The Primacy of the Ethical: Propositions for a Militant Anthropology. Current Anthropology 36(3): 409-440.

Schulte-Tenckhoff, Isabelle. 1997. La question des peuples autochtones. Paris, Bruxelles: LJDG/ Bruylant.

Schulte-Tenckhoff, Isabelle. 2012. Treaties, peoplehood and self-determination: understanding the language of Indigenous rights. In Indigenous Rights in the Age of the UN Declaration. Elvira Pulitano, eds. Pp. 64-86. Cambridge: Cambridge University Press.

Smith, Linda Tuhiwai. 1999. Decolonizing methodologies. Research and indigenous peoples. London, New York: University of Otago Press, Dunedin.

Tedlock, Barbara. 1991. From Participant Observation to the Observation of Participation: The Emergence of a Narrative Ethnography. Journal of Anthropological Research 47(1): 69-94.

Turner, Terence. 1991. Representing, Resisting, Rethinking. Historical transformations of Kayapo Culture and Anthropological Consciousness. In Colonial Situations: Essays on the Contextualization of Ethnographic knowledge. G. Stocking, eds. Pp. 285-313. Madison: University of Wisconsin Press.

Wamytan, Léon. 2013. Peuple kanak et droit français : du droit de la colonisation au droit de la décolonisation, l'égalité en question. Thèse de doctorat de droit public, Université d'Auvergne.

Wamytan, Léon. 2014. Les sources du droit de vote en Nouvelle-Calédonie : d'un droit inégalitaire à l'encontre du peuple kanak au droit inégalitaire partagé pour une citoyenneté locale. http:// www.rochwamytan.com/article-les-sources-du-droit-de-vote-en-nouvelle-caledonie-d-un-droitinegalitaire-a-l-encontre-du-peuple-122670005.html, (consulté le 31/12/2016).

Wright, Robin M. 1988. Anthropological Presuppositions of Indigenous Advocacy. Annual Review of Anthropology 17: 365-390. 


\section{ANNEXES}

ANNEXE : ÉVOLUTION DE LA POPULATION SELON LA COMMUNAUTE D'APPARTENANCE* AUX DIFFERENTS RECENSEMENTS

\begin{tabular}{|c|c|c|c|c|c|c|c|c|c|}
\hline & 1956 & 1963 & 1969 & 1976 & 1983 & 1989 & 1996 & 2009 & 2014 \\
\hline $\begin{array}{l}\text { Mélanésienne } \\
\text { (Kanak) }\end{array}$ & 34969 & 41190 & 46200 & 55598 & 61870 & 73598 & 86788 & 99078 & 104958 \\
\hline Européenne & 25160 & 33355 & 41268 & 50757 & 53974 & 55085 & 67151 & 71721 & 73199 \\
\hline $\begin{array}{l}\text { Wallisienne, } \\
\text { Futunienne }\end{array}$ & III & III & III & 9571 & 12174 & 14186 & 17763 & 21262 & 21926 \\
\hline Tahitienne & /II & III & III & 6391 & 5570 & 4750 & 5171 & 4985 & 5608 \\
\hline Indonésienne & III & III & III & 5111 & 5319 & 5191 & 5003 & 3985 & 3859 \\
\hline Vietnamienne & III & III & III & 1943 & 2381 & 2461 & 2822 & 2357 & 2506 \\
\hline Ni-Vanuatu & III & III & III & 1050 & 1212 & 1683 & 2244 & 2327 & 2568 \\
\hline Autres & 8351 & 11974 & 13111 & 2812 & 2868 & 7219 & 9894 & 39865 & 54143 \\
\hline Total & 68480 & 86519 & 100579 & 133233 & 145368 & 164173 & 196836 & 245580 & 268767 \\
\hline $\begin{array}{c}\% \text { pour la } \\
\text { population } \\
\text { kanak }\end{array}$ & $51 \%$ & $47,6 \%$ & $45,9 \%$ & $41,7 \%$ & $42,5 \%$ & $44,8 \%$ & $44 \%$ & $40,3 \%$ & $39 \%$ \\
\hline
\end{tabular}

* Avant 1976, les communautés wallisienne, tahitienne, indonésienne, vietnamienne et ni-vanuatu sont comptabilisées dans la catégorie "autres",

En 2009, sont pris en compte dans la catégorie « Autres », les autres asiatiques, plusieurs communautés minoritaires, les non-déclarés et autres.

NB : En 2004, la question sur la communauté d'appartenance n'a pas été posée.

Sources : INSEE-ISEE

L'Avenir calédonien, numéro spécial, octobre 2015

\section{NOTES}

1. Rather than the usual translation by "hut ", the author prefers to keep the French word " case»: such Kanak customary houses are places for everyday life as well as collective discussions and decisions. Their architecture reflects the structure of the whole Kanak society.

2. Stéphanie Graff fait des recherches en Nouvelle-Calédonie depuis 2005. Elle y vit depuis plusieurs années et travaille au sein d'une institution locale. Elle est la seule anthropologue à être impliquée au quotidien au cœur des sphères politiques calédoniennes notamment sur les questions qui concernent l'avenir politique et institutionnel du pays et les thématiques traitées dans sa thèse (autodétermination, décolonisation, etc.). Sa posture méthodologique dépasse celle de l'observation participante de l'ethnographie. Elle relève davantage de la « participation observante " (Tedlock 1991 ; Turner 1991 ; Albert 1997 ; Makaremi 2008) et au-delà, de la « participation radicale » (Goulet 1998 et 2004, cité dans Giabiconi 2012 : 11). Son ethnographie peut être qualifiée d'« engagée » (Wright 1988 ; Hastrup et al 1990 ; Scheper-Hugues 1995 ; Bosa 2008 ; Cefaï 2010, Giabiconi 2012), « circonstanciée » (Marcus in Cefaï 2010 : 395) et « multisituée » (Marcus 1995). Cet article se fonde sur son expérience professionnelle quotidienne. Une partie des connaissances acquises sur le terrain ne peuvent être ici utilisées ou citées par l'auteur pour des raisons de réserve et de confidentialité.

3. Les anciens combattants, chefs coutumiers, pasteurs, ou moniteurs d'enseignement kanak furent les premiers à obtenir le droit de vote en 1951, les autres l'obtinrent en 1957 (MohamedGaillard $2010: 478$ ).

4. Pour davantage de détails sur la politique de colonisation de peuplement par la France, voir Leblic 1993 ; Baissat 2006 ; Graff 2015. Voir également la Lettre de Pierre Messmer, Premier ministre, à Jean-François Deniau, secrétaire d'État aux DOM-TOM, datant du 19 juillet 1972 
(Gabriel et Kermel 1985 : 51), le tableau de l'évolution de la population de Nouvelle-Calédonie de 1887 à 1983 (Leblic 1993 : 25) et le tableau en annexe de cet article.

5. Plusieurs référendums d'autodétermination furent prévus, mais ils furent soit avortés, soit boycottés par les indépendantistes kanak en raison de leur désaccord avec la définition du corps électoral.

6. Notamment le boycott actif du statut Lemoine par les indépendantistes qui considéraient que ce nouveau statut, adopté à l'Assemblée nationale le 31 juillet 1984, ne respectait pas les négociations et accords passés durant la table ronde de Nainville-les-Roches - qui constitue la première négociation entre la France, les indépendantistes, et les non-indépendantistes notamment en ce qui concerne la définition du corps électoral. Ainsi, à Canala, le 18 novembre 1984, pour marquer ce boycott actif, Éloi Machoro brisa l'urne du bureau de vote de la mairie.

7. Les Accords de Matignon-Oudinot ont été signés le 26 juin (Accord de Matignon) et le 20 août (Accord d'Oudinot) 1988.

8. La part de la population qui sera appelée au vote est définie à l'article 218 de la loi organique $n$ - 99-209 du 19 mars 1999 relative à la Nouvelle-Calédonie, faisant suite à l'Accord de Nouméa. Cette consultation "portera [en théorie] sur le transfert à la Nouvelle-Calédonie des compétences régaliennes, l'accès à un statut international de pleine responsabilité et l'organisation de la citoyenneté en nationalité » (Point 5 de l'Accord de Nouméa).

9. Cette mission fait suite à un fort lobbying du Groupe UC-FLNKS et Nationalistes ayant dénoncé des «fraudes électorales » depuis 2013 auprès du Comité de décolonisation des Nations Unies. Il convient de préciser que cet article ne porte pas sur les enjeux actuels de l'avenir institutionnel de la Nouvelle-Calédonie, ni sur la question institutionnelle de sortie de l'Accord de Nouméa, analysés ailleurs (Graff 2015). De même, les problèmes complexes portant sur la définition des corps électoraux ne peuvent qu'être survolés ici : sur cette question et celle du droit de vote en Nouvelle-Calédonie, voir Faberon 2001; Clinchamps 2008; Chauchat 2012 ; Wamytan 2013 et 2014 ; Graff 2015.

10. Expression issue du préambule de l'Accord de Nouméa: "L'avenir doit être le temps de l'identité, dans un destin commun. "

11. Dans le contexte calédonien, si l'on se base sur les définitions des Nations Unies, déjà très largement débattues par un grand nombre de chercheurs (Daes 1986 et 1993 ; Schulte-Tenckhoff 1997 et 2012; Anaya 2000; Bowen 2000; Koubi et Schulte-Tenckhoff 2000 ; Lam 2000 ; Kuper 2003 ; Kenrick 2004; Asch 2004 et 2006; Barnard 2004 et 2006; Bellier 2013; Graff 2015), concernant les notions de "peuple autochtone» et de "peuple colonisé », le seul peuple autochtone et colonisé en Nouvelle-Calédonie est le peuple kanak. Le terme "peuple» au singulier se rapporte donc au peuple kanak. Le terme « communauté » se rapporte aux personnes issues d'autres peuples, qui, ayant émigré en Nouvelle-Calédonie et y ayant retrouvé d'autres personnes de leur peuple, ont constitué ensemble une communauté.

12. Pour une mise en perspective théorique des questions dans le cadre des controverses mémorielles en France, autour du fait colonial, voir notamment Bertrand 2006.

13. 5 jours à Nainville-les-Roches. Document du secrétariat d'Etat aux DOM-TOM, 1983.

Ci-après quelques références de déclarations faites par des présidents de la République française : François Mitterrand aux indépendantistes au moment du boycott de la loi Lemoine: «l'indépendance jamais, au pire la partition» (http://www.afriques21.org/spip.php?article11, http://madoy-nakupress.blogspot.fr/2011/01/des-bons-souvenirs-qui-restent.html, consulté le 23/09/2012) ; Nicolas Sarkozy lors de ses vœux à l'Outre-mer en 2010: "Il n'y a qu'une seule ligne rouge : celle de l'indépendance. L'outre-mer est français et restera français » (http:// infoantilles.sasi.fr/web/infoantilles/Actualites.nsf/Actualites\%20Antilles\%

20accueil/7748FFA9609ED0AE042576B000530ECB?opendocument, consulté le 30/04/2012); François Hollande aux Outre-mers en 2012 : « Et la France grâce à vous est présente partout dans le monde. Nous sommes sans doute un des rares pays de la planète à pouvoir être partout, sur 
tous les continents du monde. [...] il y a la République, la République française une, indivisible et laïque. Et la République ne craint pas la diversité [...].» (http:// www.touspourhollande.fr/2012/03/11/discours-de-francois-hollande-aux-outre-mers-dusamedi-10032012/, consulté le 23/09/2012).

14. Point 2 de la déclaration de la table ronde de Nainville-les-Roches : «[...] autodétermination ouverte également pour des raisons historiques aux autres ethnies dont la légitimité est reconnue par les représentants du peuple kanak». De plus, des représentants du RPCR, parti politique contre l'indépendance, rassemblant majoritairement la population d'ascendance européenne, furent appelés à la table des négociations au titre de "victimes de l'histoire». «Cette expression avait été avancée par les indépendantistes, notamment par l'Union Calédonienne, dès le début des années 1980, pour nommer ceux que les indépendantistes kanak acceptaient comme légitimes pour s'exprimer sur l'avenir du pays, c'est-à-dire ceux ayant subi les méfaits du colonialisme français, ceux issus d'une colonisation de peuplement forcée, ceux à qui l'administration coloniale avait imposé l'installation en Nouvelle-Calédonie » (Graff 2015 : 126).

15. Jacques Lafleur est un homme politique calédonien fondateur du Rassemblement Pour la Calédonie (RPC) en 1977 devenu un an plus tard le Rassemblement Pour la Calédonie dans la République (RPCR). Il avait décidé de fédérer tous les partisans du maintien de la NouvelleCalédonie dans la France en réponse au positionnement de l'Union Calédonienne, premier parti calédonien créé en 1956, en faveur de l'indépendance.

16. Jean-Marie Tjibaou est un homme politique kanak du parti Union Calédonienne ayant émergé sur la scène politique au travers du festival Melanesia 2000 en 1975. Il fut président de l'Union Calédonienne, et premier président du FLNKS et du gouvernement provisoire de Kanaky en 1984. Il est le signataire FLNKS des Accords de Matignon-Oudinot en 1988. Il est mort assassiné le 4 mai 1989.

17. Ancien Haut-commissaire de la République française en Nouvelle-Calédonie, impliqué dans la rédaction de l'Accord de Nouméa. Conseiller d'Etat à la retraite, Alain Christnacht est toujours engagé auprès de l'Etat sur le dossier de l'avenir institutionnel de la Nouvelle-Calédonie. Il est membre de la mission d'experts ou de «facilitateurs » en charge des "groupes de travail » et autres « comités de pilotage » notamment sur le transfert des compétences régaliennes.

18. Entretien à Paris, mars 2011.

19. Il existe en Nouvelle-Calédonie trois corps électoraux : le corps électoral permettant de voter aux élections nationales françaises et aux communales, non restreint; et deux corps électoraux restreints, pour les élections aux assemblées de province et à l'élection des membres du congrès, et pour la consultation sur l'accession du pays à la pleine souveraineté. Les critères de ces corps électoraux restreints, qui déterminent également la définition du citoyen calédonien, sont développés au point 2.2.1 de l'Accord de Nouméa et font l'objet des articles 188 et 218 de la loi organique du 19 mars 1999 relative à la Nouvelle-Calédonie.

20. Pour une meilleure idée de la répartition de la population par communauté dans Nouméa voir http://www.isee.nc/population/recensement (consulté le 31/12/2016) ainsi que le tableau en annexe.

21. Entretien avec Thierry Kameremoin, membre du «Comité 150 ans après », Nouméa, 28 octobre 2010.

22. Le Sénat coutumier est une institution créée par l'Accord de Nouméa qui fait suite au Conseil consultatif coutumier créé par les Accords de Matignon.

23. Ce comité comprenait des représentants coutumiers, des représentants de diverses associations (Association pour la Commémoration de l'Année des Peuples Indigènes en Kanaky (ACAPIK), Conseil National pour les Droits du Peuple Autochtone (CNDPA), Comité Rheebu Nuu, etc.). L'ACAPIK a été créée pour la célébration de l'année des peuples indigènes en NouvelleCalédonie. La date de l'organisation de cette célébration fut choisie pour coïncider avec la 
commémoration de « deuil kanak » de la prise de possession du 24 septembre 1853 (Monnerie 2005 : 81-118) et pour traduire en Nouvelle-Calédonie le projet de la Décennie sur les droits des peuples autochtones des Nations Unies. Le Comité Rheebù Nùù a été créé en 2002 pour contrer le projet Goro-Nickel de construction de l'usine d'exploitation de nickel dans le Sud de la NouvelleCalédonie. Avec le Congrès Populaire, créé en 1992, et le CNDPA, Conseil National des droits du peuple autochtone, épigone de l'ACAPIK, créé en 1993, ces associations constituent les fondements structurels de la revendication kanak « autochtoniste ». Leurs revendications sont en grande partie calquées sur le modèle de la Déclaration des Nations Unies sur les droits des peuples autochtones. Graff (2015) a proposé une analyse approfondie de la question hautement complexe de l'émergence d'une revendication autochtoniste à côté de la revendication indépendantiste.

24. En langue drubea, langue vernaculaire de l'aire Drubea-Kapumë du Sud de la NouvelleCalédonie.

25. Entretien avec Thierry Kameremoin, ibid. Historiquement, l'accès des Kanak à la ville était réglementé (Merle 1995). En 1975, le festival Melanésia 2000 constitue le premier grand événement public au cours duquel la culture kanak était donnée à voir, comme image kanak dans la «ville blanche ».

26. « Toutoute » est le mot communément utilisé localement pour « conque ».

27. «Il convient de faire mémoire de ces moments difficiles, de reconnaître les fautes, de restituer au peuple kanak son identité confisquée, ce qui équivaut pour lui à une reconnaissance de sa souveraineté, préalable à la fondation d'une nouvelle souveraineté, partagée dans un destin commun. " (Préambule Accord de Nouméa). Or la colonisation est-elle uniquement une perte d'identité ? N'est-elle pas aussi une perte de souveraineté... ?

28. « [...] il convient d'ouvrir une nouvelle étape, marquée par la pleine reconnaissance de l'identité kanak, préalable à la refondation d'un contrat social entre toutes les communautés qui vivent en Nouvelle-Calédonie [...]. » (Préambule Accord de Nouméa).

29. «Le moment est venu de reconnaître les ombres de la période coloniale, même si elle ne fut pas dépourvue de lumière. » (Préambule de l'Accord de Nouméa).

30. Expression utilisée communément en Nouvelle-Calédonie pour évoquer le problème de la « fête de la citoyenneté » en 2012, voir notamment Lefevre 2015.

31. Entretien avec un membre du collectif «Tribu dans la ville», puis "Ville dans la tribu», décembre 2012.

32. Il est à noter qu'au sein des représentants institutionnels kanak, les opinions étaient divergentes quant au maintien ou à la destruction des cases. Ainsi le Sénat coutumier s'était prononcé en faveur du non maintien des cases. Pour plus d'informations à ce sujet, voir l'article de Tate Lefevre déjà mentionné.

33. Pour davantage de détails sur « l'affaire des cases », voir Chauchat 2012 et Lefevre 2015. Cette dernière a analysé le "conflit dans le conflit", qui a opposé les jeunes kanak aux coutumiers kanak autour du maintien ou non des cases.

34. Selon la version officielle les hommes envoyés par la mairie étaient cagoulés pour préserver leur anonymat.

35. Radio Djiido est une radio créée par le FLNKS le 24 septembre 1985.

36. Sur les questions de «droit à la visibilité » dans l'espace géographique, voir Pantz 2015. 


\section{RÉSUMÉS}

Il existe aujourd'hui en Nouvelle-Calédonie, colonie française depuis 1853 engagée dans un processus dit de « décolonisation » depuis la signature de l'Accord de Nouméa le 5 mai 1998, une stratégie politique, que l'auteur qualifie de " politique de destin commun ». Celle-ci vise à créer un sentiment d'appartenance à une citoyenneté calédonienne grâce à la réconciliation entre les peuples et communautés en présence dans ce pays. Pour ce faire l'histoire est réinterprétée, réinventée, et réécrite en étant basée sur l'argument du destin commun, du vivre-ensemble. La réécriture de l'histoire et la réconciliation sont les moyens par lesquels l'objectif de la citoyenneté calédonienne peut être atteint. Pour illustrer cette argumentation, les exemples de la « fête de la citoyenneté » et de « l'affaire des cases » seront discutés dans cet article. Cet article s'interroge sur le sens, et l'objectif donné, des productions visuelles publiques, de même que sur ce qu'il est acceptable de voir et de montrer dans le contexte de la construction d'une citoyenneté calédonienne en Nouvelle-Calédonie.

New Caledonia has been a French colony since 1853 - it is now a sui generis overseas "collectivity" - and is currently engaged in a process of "decolonization" following the signature of the Accord de Nouméa on the $5^{\text {th }}$ of May 1998 (cf. point 4 of the preamble). This article argues that this process of decolonization is counteracted by a political strategy, which the author will refer to as the "politics of a common destiny". This strategy aims at creating a feeling of citizenship by fostering reconciliation between peoples and communities living in New Caledonia. To achieve this, history is reinterpreted and reinvented by emphasizing togetherness and shared destiny. By way of illustration, the "celebration of the citizenship" events as well as the "affaire des cases" will be here discussed. This article focuses on the meaning, and the given objective, of public visual productions, and examines what is acceptable to see and show in the context of the construction of a Caledonian citizenship in New Caledonia.

New Caledonia, decolonization, citizenship, common destiny, Mwâ Kââ, 24th September, "affaire des cases" (destruction of customary houses), tribe in the city, Noumea, Kanak presence in urban áreas.Visibilidad del destino común e invisibilidad de la historia: discurso, celebraciones y construcción de la ciudadanía en Nueva-Caledonia. Nueva Caledonia ha sido colonia francesa desde 1853 -ahora es una colectividad transoceánica sui generis- y se encuentra actualmente inmersa en un proceso de "descolonización" como consecuencia del acuerdo conocido como Accord de Nouméa firmado el 5 de mayo de 1998 (cf. Punto 4 del preámbulo). El presente artículo sostiene que este proceso de descolonización se ve contrarrestado por una estrategia política a la que el autor se referirá como "la política del destino común". Esta política tiene por objetivo crear un sentimiento de ciudadanía promoviendo la reconciliación entre los pueblos y comunidades que viven en Nueva Caledonia. Para alcanzar este objetivo, se reescribe y se reinterpreta la historia, enfatizando las ideas de comunión y porvenir compartido. A modo de ilustración, se discutirán los eventos de "la celebración de la ciudadanía", así como las "cuestión de las casas" (affaires des cases). El artículo se centra en el significado y el objetivo de producciones visuales públicas, y examina qué se acepta ver y mostrar en el contexto de la construcción de la ciudadanía de Nueva-Caledonia. 


\section{INDEX}

Keywords : New Caledonia, decolonization, citizenship, common destiny, Mwâ Kââ, 24th September, "affaire des cases" (destruction of customary houses), tribe in the city, Noumea, Kanak presence in urban areas

Mots-clés : Nouvelle-Calédonie, décolonisation, citoyenneté, destin commun, Mwâ Kââ, 24 septembre, « affaire des cases », Nouméa, présence kanak en milieu urbain

Palabras claves : Nueva Caledonia, Descolonización, ciudadanía, Destino Común, Mwâ Kââ, 24 de septiembre, cuestión de las casas (affaire des cases), tribu en la ciudad, Noumea, Presencia Kanak en las áreas urbanas

\section{AUTEUR}

\section{STÉPHANIE GRAFF}

